\title{
On hermitian-holomorphic classes related to uniformization, the dilogarithm, and the Liouville Action
}

\author{
Ettore Aldrovandi \\ Department of Mathematics \\ Florida State University \\ Tallahassee, FL 32306-4510, USA \\ aldrovandi@math.fsu.edu
}

\begin{abstract}
Metrics of constant negative curvature on a compact Riemann surface are critical points of the Liouville action functional, which in recent constructions is rigorously defined as a class in a Čech-de Rham complex with respect to a suitable covering of the surface.

We show that this class is the square of the metrized holomorphic tangent bundle in hermitian-holomorphic Deligne cohomology. We achieve this by introducing a different version of the hermitian-holomorphic Deligne complex which is nevertheless quasi-isomorphic to the one introduced by Brylinski in his construction of Quillen line bundles. We reprove the relation with the determinant of cohomology construction.

Furthermore, if we specialize the covering to the one provided by a Kleinian uniformization (thereby allowing possibly disconnected surfaces) the same class can be reinterpreted as the transgression of the regulator class expressed by the Bloch-Wigner dilogarithm.
\end{abstract}

\section{Contents}

1 Introduction 2

1.1 Organization of the paper $\ldots \ldots \ldots \ldots \ldots \ldots \ldots \ldots \ldots \ldots \ldots \ldots \ldots$

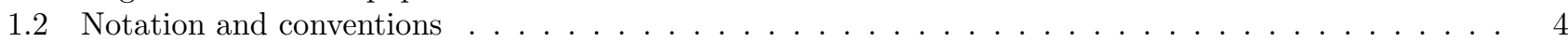

2 Deligne complexes $\quad 5$

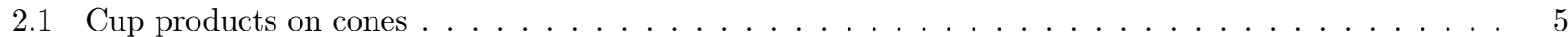

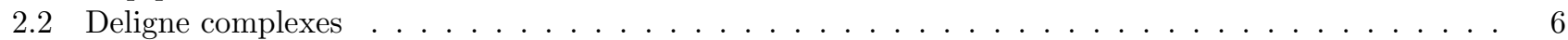

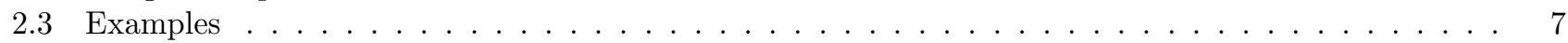

2.4 Remarks on the cup product $f \cup d \ldots \ldots \ldots \ldots \ldots \ldots \ldots$

3 Constructions in hermitian holomorphic Deligne cohomologv 9

3.1 Hermitian holomorphic Deligne cohomologv . . . . . . . . . . . . . . . . . . . . . 9 9

3.2 Hermitian holomorphic line bundles . . . . . . . . . . . . . . . . . . . . . . . . . . 11

3.3 Cup product of hermitian holomorphic line bundles . . . . . . . . . . . . . . . . . . . . 13

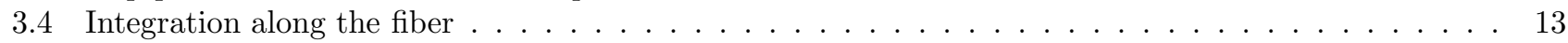

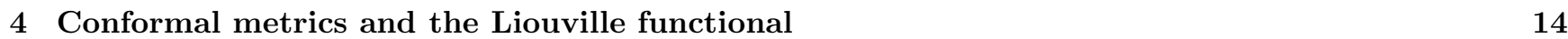

4.1 Variational problem for conformal metrics . . . . . . . . . . . . . . . . . . . . . . . . 15

4.2 Direct construction of the Liouville cocvcle $\ldots \ldots \ldots \ldots \ldots \ldots \ldots$

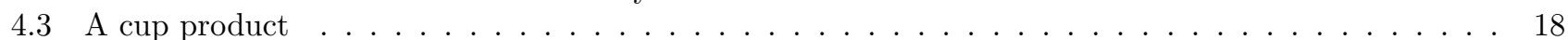

4.4 Two line bundles . . . . . . . . . . . . . . . . . . . . . . . . . . . . . . . . 18

4.5 Additional remarks on the Liouville functional and the Bloch-Wigner dilogarithm . . . . . . . . . . . 19

5 Conformal metrics and hermitian holomorphic cohomolog $\quad 20$

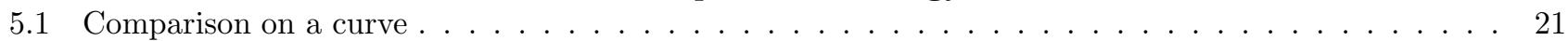

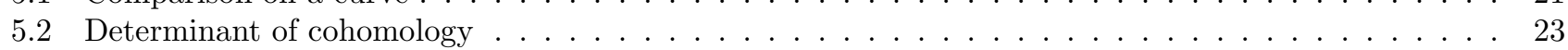

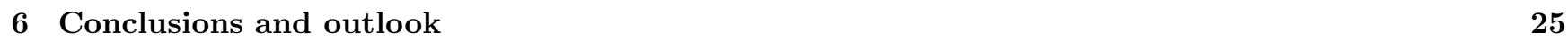


\begin{tabular}{|lr}
\hline A Cones & 25
\end{tabular}

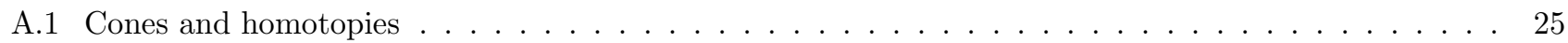

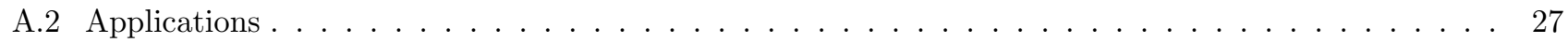

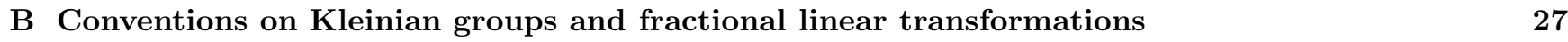

\section{Introduction}

Metrics of constant negative curvature play a very important role in uniformization problems for compact Riemann surfaces of genus $g>1$. The condition that the scalar curvature associated to a conformal metric on a Riemann surface $X$ be equal to -1 is equivalent to the fact that the associated conformal factor satisfies a nonlinear partial differential equation known as the Liouville equation.

The Liouville equation appears as early as in one of the approaches considered by Poincaré to attack the uniformization theorem [27. In relatively recent times, it has received considerable attention in Theoretical and Mathematical Physics due to the key role it plays in Polyakov's approach to String Theory [28, especially from the point of view of non-critical strings and two-dimensional quantum gravity. In this context one refers to the conformal factor of the metric as the Liouville "field."

As usual in the context of differential equations with a physical motivation, one would normally like to formulate a variational principle to express the Liouville equation as an extremum condition. Namely, given a Riemann surface $X$ and the space $\mathscr{C} \mathscr{M}(X)$ of all conformal metrics on it, the metric of constant negative curvature should be a critical point of a functional defined over $\mathscr{C} \mathscr{M}(X)$. This functional is the Liouville action. As it happens, action functionals may turn out to be even more relevant than the equations they are associated to. The Liouville action is no exception in this sense: it has deep connection with the geometry of Teichmüller spaces [33, 34, and in Physics it describes the conformal anomaly in String Theory.

Providing a rigorous mathematical definition of the Liouville action functional is however far from trivial. The very geometric properties of the Liouville field itself prevent expressing the corresponding functional as a plain integral of a 2-form on a Riemann surface. Correction terms are required, typically in the form of integration of lower degree forms over the 1-skeleton of an appropriate simplicial realization of $X$. (One should notice that this behavior is not specific to the Liouville equation, and it is by now possible to give a characterization, in terms of homological algebra, of these type of functionals, see ref. [1.)

It is possible to directly determine the necessary correction terms by requiring that the variational problem be well defined. This, however, is not completely satisfactory from the point of view of certain applications to deformation theory, where a consistent definition across a family of surfaces is required. Quite recently, a more systematic construction, based on the homological algebra techniques developed by the author and L. A. Takhtajan in 2], was carried out by L. A. Takhtajan and L.-P. Teo in ref. [30, generalizing the earlier results of [33] 34]. The authors of ref. 30] constructed a Cech cocycle with respect to the étale cover of $X$ associated to a quasi-Fuchsian (and more generally Kleinian) uniformization. Since their construction works across (Kleinian) deformations, it could be exploited to obtain results of global nature on the analytic geometry of Kleinian deformation spaces. As a further result, the authors of loc. cit. were able to rigorously prove the validity of the "holography principle" for the Liouville action corresponding to a large class of Kleinian (in particular Fuchsian and quasi-Fuchsian) uniformizations. Specifically, they proved that given a second kind Kleinian group, the corresponding Liouville action can be obtained as the regularized limit of the hyperbolic volume of the corresponding associated 3-manifold. This extends to the general Kleinian case a previous formula obtained by Krasnov 24] for classical Schottky groups.

Our interest in this matter is two-fold. From the perspective of the newer methods adopted in [3], the covering map $U \rightarrow X$ associated to the uniformization by a discrete group $\Gamma=\operatorname{Deck}(U / X)$ is but one of the many possible covers comprising an appropriate category C of, say, local diffeomorphisms $U \rightarrow X$ - the most obvious choice being that of standard open cover $\mathcal{U}=\left\{U_{i}\right\}$ with associated space $U=\coprod_{i} U_{i}$. In particular one expects to be able to apply the methods of [2] and [3] uniformly on a class of reasonably behaved covers of $X$.

Second, the focus of ref. 3] was on the rigorous definition of a functional for quasi-conformal deformation of the Riemann surface $X$ and its application to the study of projective structures. A main result is that the construction of the action is possible thanks to the vanishing of the "tame symbol" (see refs. [12] and [9] for the relevant definitions) $\left(T_{X}, T_{X}\right]$, where $T_{X}$ is the holomorphic tangent line bundle of $X$. The vanishing determines local choices (with respect to a cover) of a Bloch-type dilogarithm which then allow for a cohomological construction of the action. There are many indication that the Liouville action ought to be the hermitian square of a functional of the type studied in $3 .{ }^{3}$ Thus it is natural to ask whether there is an analogous mechanism as the one in loc. cit. to obtain

\footnotetext{
${ }^{1}$ From a physical point of view this originates in the modular geometry approach to Conformal Field Theory advocated by Friedan
} 
a general construction of the Liouville action by replacing the holomorphic symbol maps and dilogarithms with corresponding real objects.

In this paper we answer this question in the affirmative. More precisely, we show that the Liouville action (up to the area term which is given by an ordinary 2-form) can be computed as a symbol map taking values in hermitian holomorphic Deligne cohomology, first introduced by Brylinski and McLaughlin in their study of degree four characteristic classes [9]. (By way of comparison, the tame symbols used in ref. [3] used holomorphic and smooth Deligne cohomology.) In particular we show that the dilogarithm type terms are replaced here by the Bloch-Wigner function, the real valued counterpart of the dilogarithm (see refs. [7, and 23] 15] for a review.)

The appearance of the Bloch-Wigner function ties very well with the holography property of the Liouville function proved in [30] in the following sense. As mentioned before, the Liouville action (up to the area term) relative to a Kleinian uniformization ${ }^{2} U \rightarrow X$ can also be computed as the "regularized volume" of the associated 3-manifold $N=\Gamma \backslash\left(U \cup \mathbb{H}^{3}\right)$, where $\Gamma=\operatorname{Deck}(U / X)$ as before, $U \subset \mathbb{P}^{1}$ is the domain of discontinuity for $\Gamma$, and $\mathbb{H}^{3}$ is the standard hyperbolic 3 -space. (To define the regularized volume would lead us too far afield. It suffices to mention that the conformal factor of a metric on $X=\partial N$ can be used to select a compact submanifold $N_{\epsilon}$ whose volume is finite. One then subtracts from the volume of $N_{\epsilon}$ the areas of the boundary components and other carefully chosen constants independent of the metric structure, so that the resulting quantity will have a finite limit as $\epsilon \rightarrow 0$.) On the other hand, the hyperbolic volume in three dimensions corresponds to a three dimensional (purely imaginary) class on $\mathrm{PSL}_{2}(\mathbb{C})$ expressible through the Bloch-Wigner dilogarithm, the so-called regulator class. We show that the regulator is precisely the class that needs to be killed in order to close the cohomological descent conditions required to calculate the Liouville action for a covering map $U \rightarrow X$ with covering group a Kleinian group $\Gamma$. This is possible, since for a second kind Kleinian group the quotient $\mathbb{H}^{3} / \Gamma$ is non-compact, hence it carries no cohomology in dimension three, so the class represented by the Bloch-Wigner function, pulled back to $\Gamma$ via the imbedding $\Gamma \hookrightarrow \mathrm{PSL}_{2}(\mathbb{C})$, vanishes.

Returning to the cohomological interpretation of the construction of the Liouville action, it should also be noted that leaving aside the area term, our results show that the cohomologically non trivial part is indeed a square. Namely, for a conformal metric $\rho \in \mathscr{C} \mathscr{M}(X)$ we consider the pair $\left(T_{X}, \rho\right)$ as a holomorphic line bundle equipped with an hermitian metric. Then, using that hermitian holomorphic Deligne cohomology has a cup product, we show that the Liouville action is just the square of the class of $\left(T_{X}, \rho\right)$. In fact this identification holds at the level of cocycles, rather than only for the corresponding classes.

Again leaving aside the area term, it immediately follows from the properties of hermitian holomorphic Deligne cohomology that most of the story carries over to the case of a pair of holomorphic line bundles $L$ and $L^{\prime}$ equipped with hermitian metrics $\rho$ and $\rho^{\prime}$, respectively. Furthermore, Brylinski shows in [10] that the pairing of two such holomorphic line bundles with metrics corresponds to the pairing defined by Deligne on the determinant of cohomology in 11. Without introducing the machinery of 2-gerbes, we reobtain this result in our setting. Specifically, we directly obtain Gabber's formula for the hermitian metric on the determinant line from the explicit cocycle for the cup product of two metrized line bundles. In turn this shows that the Liouville action is a multiple of the determinant of cohomology, thereby generalizing earlier results (cf. 32] —without assuming criticality.

\subsection{Organization of the paper}

This paper is organized as follows. Sections 2 and 3 are devoted to expounding some background material for the sake of keeping this paper self-contained and to put the reader in position of reproducing the necessary calculations. Section 2 contains background facts on Deligne cohomology, paying special attention to the product structures and the cone constructions. We provide some examples and collect some facts about the dilogarithm from the point of view of Deligne cohomology. The particular model of hermitian holomorphic Deligne cohomology we use later in the paper requires certain constructions available in the literature, and recalled in section 2 to be slightly modified in order to obtain a (graded) commutative product. The necessary arguments, being somewhat outside the line of development of the paper are presented in Appendix A Hermitian holomorphic Deligne cohomology is introduced in section [3. We give the definition as in refs. 9] and [10, and then introduce another model which, albeit more complex, has the advantage for us of keeping the metric structure explicit. We have explicitly proved the isomorphism in Lemma 3.2 For the sake of completeness, we give an explicit description of the cocycle determined by a holomorphic line bundle with hermitian metric, and in sect. 3.3 we explicitly compute the cup product of two metrized line bundles for later usage. Results about the existence of a fiber integration map are mentioned in the paper, so some background material is provided in sect. 3.4.

and Shenker in [19]. Mathematically speaking, it is one of the many proposed forms of the holomorphic factorization property for determinant line bundles.

${ }^{2}$ Note that $X$ is allowed to be disconnected. 
Sections 4 and 5 form the core of the paper. The direct construction of the Liouville action according to the techniques of refs. 2, 3, 30, is presented in section 4, Since explicit calculations have been presented in great detail in ref. [30, and the calculations we need are quite straightforward, we keep details to a minimum. In Proposition 4.4 and Corollary 4.5 we show that the Liouville action functional computed via descent theory does solve the variational problem. These results have appeared also in ref. 30] and are presented for here completeness, although our choice for the cover is different. The framework of loc. cit. is that of a Kleinian cover $U \rightarrow X \cong U / \Gamma$, where $\Gamma$ is a second kind purely loxodromic geometrically finite Kleinian group, which then we treat in some detail in sect. 4.5. We illustrate how the genuine Bloch-Wigner function of sect. 2.4 appears in the descent equations relative to the Kleinian cover. Having observed descent equations close on general cohomological grounds in sect. 4.2.3 we now point out that for the case of a Kleinian cover this is due to the vanishing of the regulator class for the non-compact 3-manifold $\mathbb{H}^{3} / \Gamma$.

A true geometric construction of the Liouville action, which does not rely on the arguments of sect. 4 to close the descent equations, is carried out in section 5 which contains our main result: We compare the descent calculations with the cup products computed in section 3 and conclude that the quadratic part of the Liouville action is in fact (modulo an area term) the cup square of the metrized holomorphic tangent bundle in Hermitian holomorphic Deligne cohomology, see Theorem [5.1] Corollary 5.2] and Proposition[5.4 It follows that from this point of view the descent equations close automatically, without the need for special arguments. We then prove that the cup square is identified with the determinant of cohomology construction in Theorem [5.5] and Corollary [5.6 Auxiliary facts about the homological algebra of cones and conventions on Kleinian groups are stored in the appendices.

Finally, we draw our conclusions in sect. [6]

\section{$1.2 \quad$ Notation and conventions}

If $z$ is a complex number, then $\pi_{p}(z) \stackrel{\text { def }}{=} \frac{1}{2}\left(z+(-1)^{p} \bar{z}\right)$, and similarly for any other complex quantity, e.g. complex valued differential forms. If $A$ is a subring of $\mathbb{R}$, we will use the "twist" $A(j)=(2 \pi \sqrt{-1})^{j} A$.

If $X$ is a complex manifold, $\underline{A}_{X}^{\bullet}$ and $\underline{\Omega}_{X}^{\bullet}$ denote the de Rham complexes of smooth $\mathbb{C}$-valued and holomorphic forms, respectively. We denote by $\underline{\varepsilon}_{X}^{\bullet}$ the de Rham complex of sheaves of real valued differential forms and by $\underline{\varepsilon}_{X}^{\bullet}(j)$ the twist $\underline{\varepsilon}_{X}^{\bullet} \otimes_{\mathbb{R}} \mathbb{R}(j)$. We set $\mathcal{O}_{X} \equiv \underline{\Omega}_{X}^{0}$ as usual. When needed, $\underline{A}_{X}^{p, q}$ will denote the sheaf of smooth $(p, q)$-forms. We use the standard decomposition $d=\partial+\bar{\partial}$ according to types. Furthermore, we introduce the differential operator $d^{c}=\partial-\bar{\partial}$ (contrary to the convention, see, e.g. [25], we omit the factor $1 /(4 \pi \sqrt{-1})$ ). We have $2 \partial \bar{\partial}=d^{c} d$. The operator $d^{c}$ is an imaginary one, and accordingly, we have the rules

$$
\begin{gathered}
d \pi_{p}(\omega)=\pi_{p}(d \omega) \\
d^{c} \pi_{p}(\omega)=\pi_{p+1}\left(d^{c} \omega\right)
\end{gathered}
$$

for any complex form $\omega$.

An open cover of $X$ will be denoted by $\mathcal{U}_{X}$. If $\left\{U_{i}\right\}_{i \in I}$ is the corresponding collection of open sets, we denote $U_{i j}=U_{i} \cap U_{j}, U_{i j k}=U_{i} \cap U_{j} \cap U_{k}$, and so on. We can also consider more general covers $\mathcal{U}_{X}=\left\{U_{i} \rightarrow X\right\}_{i \in I}$ where the maps are regular coverings in the appropriate category. In this case intersections are replaced by $(n+1)$-fold fibered products $U_{i_{0} i_{1} \ldots i_{n}}=U_{i_{0}} \times_{X} \cdots \times_{X} U_{i_{n}}$. Open coverings fit this more general description, since if $U_{i}$ and $U_{j}$ are two open sets, then $U_{i} \cap U_{j}=U_{i} \times_{X} U_{j}$, where the fiber product is taken with respect to the inclusion maps. As another example, one can consider regular covering maps $U \rightarrow X$ with $\Gamma=\operatorname{Deck}(U / X)$, and in this case, taking the fiber product over $X(n+1)$-times, one gets $U \times_{X} \cdots \times_{X} U=U \times \Gamma \times \cdots \times \Gamma$, where the group factor is repeated $n$-times. This includes the cases of Kleinian (and in particular Fuchsian) covers of Riemann surfaces.

The nerve of the cover $\mathcal{U}_{X}$ is the simplicial object $n \mapsto N_{n}\left(\mathcal{U}_{X}\right)=\coprod U_{i_{0}} \times_{X} \cdots \times_{X} U_{i_{n}}$ where $N_{n}\left(\mathcal{U}_{X}\right)$ maps into $N_{n-1}\left(\mathcal{U}_{X}\right)$ in $(n+1)$ ways by forgetting in turn each factor. For open covers this just yields the expected inclusion maps.

If $\underline{F}^{\bullet}$ is a complex of sheaves on $X$, its Čech resolution with respect to a covering $\mathcal{U}_{X} \rightarrow X$ is the double complex $C^{p, q}(\underline{F}) \stackrel{\text { def }}{=} \check{C}^{q}\left(\mathcal{U}_{X}, \underline{F}^{p}\right)$, where the $q$-cochains with values in $\underline{F}^{p}$ are given by $\prod \underline{F}^{p}\left(U_{i_{0}} \times_{X} \cdots \times_{X} U_{i_{n}}\right)$. The Cech coboundary operator is denoted $\check{\delta}$. The sign convention we are going to use is that the index along the Cech resolution is the second one, so if we denote by $d$ the differential in the complex $\underline{F}^{\bullet}$, the total differential in the total simple complex of $\check{C}^{q}\left(\mathcal{U}_{X}, \underline{F}^{p}\right)$ will be $D=d \pm \check{\delta}$. For open covers we just get the familiar Čech (hyper)cohomology. The other interesting example is that of a regular covering map $U \rightarrow X$ : Cech cohomology with respect to this cover is the same as group cohomology for $\Gamma=\operatorname{Deck}(U / X)$ with coefficients in the $\Gamma$-module $\underline{F}^{p}(U)$.

The Koszul sign rule that results in a sign being picked whenever two degree indices are formally exchanged is applied. In particular, for Čech resolutions of complexes of sheaves, it leads to the following conventions. If $\underline{G}^{\bullet}$ is 
a second complex of sheaves on $X$, then one defines the cup product

$$
\cup: C^{p, q}(\underline{F}) \otimes C^{r, s}(\underline{G}) \longrightarrow \check{C}^{q+s}\left(\mathcal{U}_{X}, \underline{F}^{p} \otimes \underline{G}^{r}\right) \subset C^{p+r, q+s}(\underline{F} \otimes \underline{G})
$$

of two elements $\left\{f_{i_{0}, \ldots, i_{q}}\right\} \in \mathrm{C}^{p, q}(\underline{F})$ and $\left\{g_{j_{0}, \ldots, j_{s}}\right\} \in \mathrm{C}^{r, s}(\underline{G})$ by

$$
(-1)^{q r} f_{i_{0}, \ldots, i_{q}} \otimes g_{i_{q}, i_{q+1}, \ldots, i_{q+s}} .
$$

\section{Acknowledgments}

Parts of this work were completed during visits at the International School for Advanced Studies (SISSA) in Trieste, Italy, and at the Department of Mathematics, Instituto Superior Técnico in Lisbon, Portugal. I would like to thank both institutions for support and for creating a friendly and stimulating research environment. I would also like to thank Paolo Aluffi, Phil Bowers, Ugo Bruzzo, Johan Dupont, Leon Takhtajan for illuminating discussions and/or patiently answering my many questions. Also, special thanks are due to the referee for his or her thoroughness and for providing very detailed comments.

\section{Deligne complexes}

\subsection{Cup products on cones}

Recall that the cone of a map $f: X^{\bullet} \rightarrow Y^{\bullet}$ between two complexes is the complex $C^{\bullet}(f)=X^{\bullet}[1] \oplus Y^{\bullet}$ with differential $d_{f}(x, y)=(-d x, f(x)+d y)$, where $[k]$ denotes the shift functor. The cone fits into the exact sequence

$$
0 \longrightarrow Y^{\bullet} \longrightarrow C^{\bullet}(f) \longrightarrow X^{\bullet}[1] \longrightarrow 0 \text {. }
$$

The following constructions are a special case of those considered by Beĭlinson in ref. [5]. Suppose we are given complexes $X_{i}^{\bullet}, Y_{i}^{\bullet}$, and $Z_{i}^{\bullet}$ and maps $f_{i}: X_{i}^{\bullet} \rightarrow Z_{i}^{\bullet}, g_{i}: Y_{i}^{\bullet} \rightarrow Z_{i}^{\bullet}$, for $i=1,2,3$. Suppose also that we have product maps $X_{1}^{\bullet} \otimes X_{2}^{\bullet} \stackrel{\cup}{\rightarrow} X_{3}^{\bullet}$, and similarly for $Y_{i}^{\bullet}$, and $Z_{i}^{\bullet}$, strictly compatible with the $f_{i}, g_{i}$ in the obvious sense. Then we can consider the cones

$$
\text { Cone }\left(f_{i}-g_{i}\right)[-1] \equiv \text { Cone }\left(X_{i}^{\bullet} \oplus Y_{i}^{\bullet} \stackrel{f_{i}-g_{i}}{\longrightarrow} Z_{i}^{\bullet}\right)[-1] .
$$

For a real parameter $\alpha$, there is a family of products

$$
\text { Cone }\left(f_{1}-g_{1}\right)[-1] \otimes \text { Cone }\left(f_{2}-g_{2}\right)[-1] \stackrel{\cup_{\alpha}}{\longrightarrow} \text { Cone }\left(f_{3}-g_{3}\right)[-1]
$$

determined as follows. For $\left(x_{i}, y_{i}, z_{i}\right) \in X_{i}^{\bullet} \oplus Y_{i}^{\bullet} \oplus Z_{i}^{\bullet-1}, i=1,2$, one defines

$$
\begin{aligned}
\left(x_{1}, y_{1}, z_{1}\right) \cup_{\alpha}\left(x_{2}, y_{2}, z_{2}\right)= & \left(x_{1} \cup x_{2}, y_{1} \cup y_{2},\right. \\
& (-1)^{\operatorname{deg}\left(x_{1}\right)}\left((1-\alpha) f_{1}\left(x_{1}\right)+\alpha g_{1}\left(y_{1}\right)\right) \cup z_{2} \\
& \left.+z_{1} \cup\left(\alpha f_{2}\left(x_{2}\right)+(1-\alpha) g_{2}\left(y_{2}\right)\right)\right) .
\end{aligned}
$$

Note that $\operatorname{deg}\left(x_{1}\right)=\operatorname{deg}\left(x_{1}, y_{1}, z_{1}\right)$. Checking that $\cup_{\alpha}$ is a map of complexes is a straightforward routine calculation. Different products for different values $\alpha$ and $\beta$ of the real parameter are homotopic. Explicitly, we have

$$
\left(x_{1}, y_{1}, z_{1}\right) \cup_{\alpha}\left(x_{2}, y_{2}, z_{2}\right)-\left(x_{1}, y_{1}, z_{1}\right) \cup_{\beta}\left(x_{2}, y_{2}, z_{2}\right)=\left(d h_{\alpha, \beta}+h_{\alpha, \beta} d\right)\left(\left(x_{1}, y_{1}, z_{1}\right) \otimes\left(x_{2}, y_{2}, z_{2}\right)\right),
$$

where the homotopy

$$
h_{\alpha, \beta}: \operatorname{Tot}\left(\text { Cone }\left(f_{1}-g_{1}\right)[-1] \otimes \operatorname{Cone}\left(f_{2}-g_{2}\right)[-1]\right)^{\bullet} \longrightarrow \text { Cone }\left(f_{3}-g_{3}\right)[-1]^{\bullet-1}
$$

is given by the formula

$$
h_{\alpha, \beta}\left(\left(x_{1}, y_{1}, z_{1}\right) \otimes\left(x_{2}, y_{2}, z_{2}\right)\right)=(\alpha-\beta)(-1)^{\operatorname{deg}\left(x_{1}\right)-1}\left(0,0, z_{1} \cup z_{2}\right) .
$$


If the products $X_{1}^{\bullet} \otimes X_{2}^{\bullet} \stackrel{\cup}{\rightarrow} X_{3}^{\bullet}$, etc., are graded commutative, then the swap functor on the tensor product maps the $\cup_{\alpha}$ product structure on the cones into the $\cup_{1-\alpha}$ structure. Using the homotopy (2.3) it follows at once that there is a well defined graded commutative product in cohomology.

If we do not assume the product structures $X_{1}^{\bullet} \otimes X_{2}^{\bullet} \stackrel{\cup}{\rightarrow} X_{3}^{\bullet}$, etc., are strictly compatible with the maps $f_{i}$, $g_{i}$, some of the preceding constructions must be slightly modified. With an eye toward certain constructions to be carried out later in this paper, let us assume we have compatibility up to homotopy, namely there exist maps

$$
\begin{gathered}
h:\left(X_{1} \otimes X_{2}\right)^{\bullet} \longrightarrow Z_{3}^{\bullet-1} \\
k:\left(Y_{1} \otimes Y_{2}\right)^{\bullet} \longrightarrow Z_{3}^{\bullet-1}
\end{gathered}
$$

such that

$$
\begin{gathered}
f_{3} \circ \cup-\cup \circ\left(f_{1} \otimes f_{2}\right)=d h+h d \\
g_{3} \circ \cup-\cup \circ\left(g_{1} \otimes g_{2}\right)=d k+k d,
\end{gathered}
$$

with obvious meaning of the symbols.

Lemma 2.1. Let $X_{i}, Y_{i}, Z_{i}$ and the maps $f_{i}, g_{i}$ be as above. Let $\alpha$ be a real parameter. We have a product of type (2.1) for the cones Cone $\left(f_{i}-g_{i}\right)[-1]$ defined by the following modification of formula (2.2):

$$
\begin{aligned}
&\left(x_{1}, y_{1}, z_{1}\right) \cup_{\alpha}\left(x_{2}, y_{2}, z_{2}\right)=( x_{1} \cup x_{2}, y_{1} \cup y_{2}, \\
&(-1)^{\operatorname{deg}\left(x_{1}\right)}\left((1-\alpha) f_{1}\left(x_{1}\right)+\alpha g_{1}\left(y_{1}\right)\right) \cup z_{2} \\
&+z_{1} \cup\left(\alpha f_{2}\left(x_{2}\right)+(1-\alpha) g_{2}\left(y_{2}\right)\right) \\
&\left.-h\left(x_{1} \otimes x_{2}\right)+k\left(y_{1} \otimes y_{2}\right)\right) .
\end{aligned}
$$

The product (2.5) is a map of complexes and two products $\cup_{\alpha}$ and $\cup_{\beta}$ are related by the same homotopy formula (2.3).

Proof. Direct verification.

This modified framework carries over to the full structure considered by Beilinson in ref. [5]. We will still refer to this modified product as the Bellinson product. It is also necessary to relax the assumption that the products $X_{1}^{\bullet} \otimes X_{2}^{\bullet} \stackrel{\cup}{\rightarrow} X_{3}^{\bullet}$, etc., be graded commutative. It is possible to complete all the diagrams so that the permutation of factors in the tensor products still yields a homotopy commutative product (2.5) for the cones. As a consequence the induced product in cohomology will still be graded commutative. Explicit formulas are not needed except to ensure this latter fact, therefore we shall not discuss this matter any further and refer the reader to the appendix, where a brief but explicit treatment can be found.

\subsection{Deligne complexes}

Let $X$ be a complex manifold. Recall the standard Hodge filtration of $\underline{\Omega}_{X}^{\bullet}$ :

$$
F^{p} \underline{\Omega}_{X}^{\bullet}: 0 \longrightarrow \cdots \longrightarrow \underline{\Omega}_{X}^{p} \longrightarrow \cdots \longrightarrow \underline{\Omega}_{X}^{n},
$$

where $n=\operatorname{dim}_{\mathbb{C}} X$.

The corresponding filtration for the complex of smooth $\mathbb{C}$-valued forms is defined as follows: denote by $F^{p} \underline{A}_{X}^{\bullet}$ the subcomplex of $\underline{A}_{X}^{\bullet}$ comprising forms of type $(r, s)$ where $r$ is at least $p$, so that $F^{p} \underline{A}_{X}^{k}=\oplus_{r \geq p} \underline{A}_{X}^{r, k-r}$. Then (cf. [13]) the inclusion $\underline{\Omega}_{X}^{\bullet} \hookrightarrow \underline{A}_{X}^{\bullet}$ is a quasi-isomorphism respecting the filtrations, namely $F^{p} \underline{\Omega}_{X}^{\bullet} \hookrightarrow F^{p} \underline{A}_{X}^{\bullet}$, and the latter inclusion induces an isomorphism in cohomology.

If $A$ is a subring of $\mathbb{R}$, and $\imath$ and $\jmath$ denote the inclusions of $A(p)$ and $F^{p} \underline{\Omega}_{X}^{\bullet}$ into $\underline{\Omega}_{X}^{\bullet}$ respectively, the $p$-th Deligne complex of sheaves is defined by

$$
A(p)_{\mathcal{D}}^{\bullet}=\operatorname{Cone}\left(A(p)_{X} \oplus F^{p} \underline{\Omega}_{X}^{\bullet} \stackrel{\imath-\jmath}{\longrightarrow} \underline{\Omega}_{X}^{\bullet}\right)[-1] .
$$

It is quasi-isomorphic to the complex:

$$
\text { Cone }\left(A(p)_{X} \oplus F^{p} \underline{A}_{X}^{\bullet} \stackrel{\imath-\jmath}{\longrightarrow} \underline{A}_{X}^{\bullet}\right)[-1]
$$


where $\imath$ and $\jmath$ have the same meaning. We also notice the quasi-isomorphism

$$
A(p)_{\mathcal{D}}^{\bullet \stackrel{\simeq}{\longrightarrow}}\left(A(p)_{X} \stackrel{\imath}{\longrightarrow} \mathcal{O}_{X} \stackrel{d}{\longrightarrow} \underline{\Omega}_{X}^{1} \stackrel{d}{\longrightarrow} \cdots \stackrel{d}{\longrightarrow} \underline{\Omega}_{X}^{p-1}\right) .
$$

When $A=\mathbb{R}$ there are further quasi-isomorphisms, namely

$$
\mathbb{R}(p)_{\mathcal{D}}^{\bullet} \stackrel{\simeq}{\rightarrow} \text { Cone }\left(F^{p} \underline{\Omega}_{X}^{\bullet} \rightarrow \underline{\mathcal{E}}_{X}^{\bullet}(p-1)\right)[-1] \stackrel{\simeq}{\rightarrow} \text { Cone }\left(F^{p} \underline{A}_{X}^{\bullet} \rightarrow \underline{\mathcal{E}}_{X}^{\bullet}(p-1)\right)[-1]
$$

since the maps

$$
\left(\mathbb{R}(p) \rightarrow \underline{\Omega}_{X}^{\bullet}\right) \stackrel{\simeq}{\rightarrow}(\mathbb{R}(p) \rightarrow \mathbb{C}) \stackrel{\simeq}{\rightarrow} \mathbb{R}(p-1) \stackrel{\simeq}{\rightarrow} \underline{\varepsilon}_{X}^{\bullet}(p-1)
$$

are all quasi-isomorphisms in the derived category, cf. [18. Here we have used $\mathbb{C} \cong \mathbb{R}(p) \oplus \mathbb{R}(p-1)$. Following op. cit., we set:

$$
\widetilde{\mathbb{R}(p)})_{\mathcal{D}}^{\bullet} \stackrel{\text { def }}{=} \text { Cone }\left(F^{p} \underline{A}_{X}^{\bullet} \stackrel{-\pi_{p-1}}{\longrightarrow} \underline{\varepsilon}_{X}^{\bullet}(p-1)\right)[-1] .
$$

Again, there is an explicit quasi-isomorphism (4, 18]):

$$
\begin{gathered}
\rho_{p}: \mathbb{R}(p)_{\mathcal{D}}^{\bullet} \stackrel{\simeq}{\longrightarrow} \widetilde{\mathbb{R}(p)_{\mathcal{D}}^{\bullet}} \\
\left.\rho_{p}\right|_{\mathbb{R}(p)=0,\left.\quad \rho_{p}\right|_{F^{p}} \underline{\Omega}_{X}^{\bullet}}=\text { incl },\left.\quad \rho_{p}\right|_{\underline{\Omega}_{X}^{\bullet}}=\pi_{p-1}
\end{gathered}
$$

The Deligne cohomology groups of $X$ with coefficients in $A(p)$ are the hypercohomology groups

$$
H_{\mathcal{D}}^{\bullet}(X, A(p))=\mathbf{H}^{\bullet}\left(X, A(p)_{\mathcal{D}}^{\bullet}\right) .
$$

and clearly, any complex quasi-isomorphic to $A(p)_{\mathcal{D}}^{\bullet}$ would do. In order to perform calculations with these cohomology groups we shall normally resort to a Cech resolution with respect to an open cover $\mathcal{U}_{X}$ of $X$ or an étale map $\mathcal{U}_{X} \rightarrow X$, e.g. a regular cover with deck group $\Gamma$.

One of the important properties of Deligne cohomology is the existence of a graded commutative cup product

$$
H_{\mathcal{D}}^{i}(X, A(p)) \otimes H_{\mathcal{D}}^{j}(X, A(q)) \stackrel{\cup}{\rightarrow} H_{\mathcal{D}}^{i+j}(X, A(p+q)),
$$

which follows from the existence of the Beilinson cup product at the level of Deligne complexes whose construction was recalled above. There are products $A(p) \otimes A(q) \rightarrow A(p+q)$ and $F^{p} \underline{\Omega}_{X}^{\bullet} \otimes F^{q} \underline{\Omega}_{X}^{\bullet} \rightarrow F^{p+q} \underline{\Omega}_{X}^{\bullet}$, plus the obvious (wedge) product on $\underline{\Omega}_{X}^{\bullet}$, thus it follows from the cone version (2.7) that the Deligne complexes come equipped with the Beilinson product, and therefore the Deligne cohomology groups inherit the graded commutative cup product (2.12). The explicit form, that is, the translation of (2.2) to the case at hand can be found in 18. The explicit form of the cup product for the complex (2.10) as computed in [4 (see also [18) will be needed in the sequel. Let $\left(\omega_{1}, \eta_{1}\right)$ be an element of degree $n$ in $\widehat{\mathbb{R}}(p)_{\mathcal{D}}^{\bullet}$-this means that $\omega_{1} \in F^{p} \underline{\Omega}_{X}^{n}$ and $\eta_{1} \in \underline{\mathcal{E}}_{X}^{n-1}(p-1)$-and $\left(\omega_{2}, \eta_{2}\right)$ any element in $\mathbb{R}(q)_{\mathcal{D}}^{\bullet}$. The product is defined by the formula:

$$
\left(\omega_{1}, \eta_{1}\right) \tilde{\cup}\left(\omega_{2}, \eta_{2}\right)=\left(\omega_{1} \wedge \omega_{2},(-1)^{n} \pi_{p} \omega_{1} \wedge \eta_{2}+\eta_{1} \wedge \pi_{q} \omega_{2}\right) .
$$

The product $\tilde{U}$ is a morphism of complexes and (modulo the quasi-isomorphisms $\rho_{p}$ ) is homotopic to the Beilinson products $\cup_{\alpha}$ on the complexes $\mathbb{R}(p)_{\mathcal{D}}^{\bullet}$. Specifically, if we denote an element of $\mathbb{R}(p)_{\mathcal{D}}^{\bullet}$ by the triple $(r, f, \omega)$, where $r \in \mathbb{R}(p)_{X}, f \in F^{p} \underline{\Omega}_{X}^{\bullet}$, and $\omega \in \underline{\Omega}_{X}^{\bullet}$, the homotopy between $\tilde{\cup} \circ\left(\rho_{p} \otimes \rho_{q}\right)$ and $\rho_{p+q} \circ \cup_{\alpha}$ is given by

$$
\tilde{h}\left((r, f, \omega) \otimes\left(r^{\prime}, f^{\prime}, \omega^{\prime}\right)\right)=(-1)^{\operatorname{deg} \omega}\left(0,(1-\alpha) \pi_{p} \omega \wedge \pi_{q-1} \omega^{\prime}-\alpha \pi_{p-1} \omega \wedge \pi_{q} \omega^{\prime}\right) .
$$

\subsection{Examples}

\subsection{1}

Let $A=\mathbb{Z}$. From (2.9) we have $\mathbb{Z}(1)_{\mathcal{D}}^{\bullet} \stackrel{\simeq}{\rightarrow} \mathcal{O}_{X}^{\times}[-1]$ via the standard exponential sequence, so that $H_{\mathcal{D}}^{k}(X, \mathbb{Z}(1)) \cong$ $H^{k-1}\left(X, \mathcal{O}_{X}^{\times}\right)$. In particular $H_{\mathcal{D}}^{1}(X, \mathbb{Z}(1)) \cong H^{0}\left(X, \mathcal{O}_{X}^{\times}\right)$, the global invertibles on $X$, and $H_{\mathcal{D}}^{2}(X, \mathbb{Z}(1)) \cong \operatorname{Pic}(X)$, the Picard group of line bundles over $X$.

If an open cover $\left\{U_{i}\right\}_{i \in I}$ of $X$ is chosen, the class of a line bundle $L$ in $H_{\mathcal{D}}^{2}(X, \mathbb{Z}(1))$ can be represented via a Čech resolution by the cocycle $\left(f_{i j}, c_{i j k}\right)$, where $f_{i j} \in \mathcal{O}_{X}\left(U_{i j}\right)$, and $c_{i j k} \in \mathbb{Z}(1)_{X}\left(U_{i j k}\right)$. Thus the functions $f_{i j}$ should be interpreted as logarithms of the corresponding transition functions for $L$. Then, the collection $c_{i j k}=(\check{\delta} f)_{i j k}$ provides a representative for the first Chern Class $c_{1}(L)$. Similarly, an invertible function $f$ would be described by the collection $f_{i}$ of its logarithms on each open $U_{i}$, plus the "integers" $m_{i j}=f_{j}-f_{i} \in \mathbb{Z}(1)$ on each $U_{i j}$. 


\subsection{2}

Still using the exponential sequence, $\mathbb{Z}(2)_{\mathcal{D}}^{\bullet} \stackrel{\simeq}{\rightarrow}\left(\mathcal{O}_{X}^{\times} \stackrel{d \log }{\longrightarrow} \underline{\Omega}_{X}^{1}\right)[-1]$. Thus $H_{\mathcal{D}}^{2}(X, \mathbb{Z}(2))$ is the group of isomorphism classes of holomorphic line bundles with (holomorphic) connection. Using the (in fact, any) product $\mathbb{Z}(1)_{\mathcal{D}}^{\bullet} \otimes$ $\mathbb{Z}(1)_{\mathcal{D}}^{\bullet} \rightarrow \mathbb{Z}(2)_{\mathcal{D}}^{\bullet}$, the cup product of two global invertible holomorphic functions $f$ and $g$ on $X$ yields a line bundle with connection - the tame symbol - denoted by $(f, g]$ whose class is in $H_{\mathcal{D}}^{2}(X, \mathbb{Z}(2))$, see [12, 6] 29]. Higher cup products in this spirit have been studied in [9].

\subsection{3}

If $A=\mathbb{R}$, we have $H_{\mathcal{D}}^{2 p}(X, \mathbb{R}(p))=H^{2 p}(X, \mathbb{R}(p)) \cap H^{p, p}(X)$. The Čech representative $\left(c_{i j k}, f_{i j}\right)$ of a class in $H_{\mathcal{D}}^{2}(X, \mathbb{Z}(1))$ mentioned above maps to the cocycle $\left(-d f_{i j},-\left|f_{i j}\right|\right)$ under 2.11). Taking into account that the $f_{i j}$ are the logarithms of the transition functions, the corresponding $(1,1)$ class would be given by the associated canonical connection, see sec. 3.2

\subsection{4}

$H_{\mathcal{D}}^{1}(X, \mathbb{R}(1))$ is the group of real valued functions $f$ on $X$ such that there exists a holomorphic one-form $\omega$ such that $\pi_{0} \omega=d f$. In other words it is the group of those smooth functions $f$ such that $\partial f$ is holomorphic, which amounts to say that such an $f$ itself is harmonic.

\subsection{Remarks on the cup product $f \cup g$}

It is convenient to consider the case of the cup product of two invertible functions $f$ and $g$ in various complexes in more detail, and to introduce some related notions we shall need later.

As observed, $\mathbb{Z}(1)_{\mathcal{D}}^{\bullet} \stackrel{\simeq}{\rightarrow} \mathcal{O}_{X}^{\times}[-1]$ and an invertible function $f$ can be considered as an element of $H_{\mathcal{D}}^{1}(X, \mathbb{Z}(1))$. Therefore, via (2.11), it induces $\rho_{1}(f) \in H_{\mathcal{D}}^{1}(X, \mathbb{R}(1))$ represented by $(d \log f, \log |f|)$. (Note that $\log |f|$ fits the description of $H_{\mathcal{D}}^{1}(X, \mathbb{R}(1))$ in 2.3.4) Given two such $f$ and $g$, the expression for the cup product (2.13) gives the following element of $H_{\mathcal{D}}^{2}(X, \mathbb{R}(2))$ :

$$
\rho_{1}(f) \tilde{\cup} \rho_{1}(g)=\left(d \log f \wedge d \log g,-\pi_{1}(d \log f) \log |g|+\log |f| \pi_{1}(d \log g)\right) .
$$

The first term is obviously zero when $X$ is a curve. Given $f$ and $g$, invertible on $X$, let us define the imaginary 1-form:

$$
r_{2}(f, g)=\pi_{1}(d \log f) \log |g|-\log |f| \pi_{1}(d \log g) .
$$

On the other hand, the cup product of $f$ and $g$ as elements of $H_{\mathcal{D}}^{1}(X, \mathbb{Z}(1))$ yields an element $f \cup g$ of $H_{\mathcal{D}}^{2}(X, \mathbb{Z}(2))$ represented by $(d \log f \wedge d \log g, \log f d \log g)$ (if we use the $\cup_{0}$ product) and this maps via $\rho_{2}$ to the element

$$
\left(d \log f \wedge d \log g,-\pi_{1}(\log f d \log g)\right) .
$$

This is equal to (2.16) only up to homotopy. Indeed, using $\pi_{p+q-1}(a \wedge b)=\pi_{p}(a) \wedge \pi_{q-1}(b)+\pi_{p-1}(a) \wedge \pi_{q}(b)$, we find

$$
r_{2}(f, g)=d\left(\pi_{1}(\log f) \log |g|\right)-\pi_{1}(\log f d \log g),
$$

where the first term is just the explicit homotopy as computed from (2.14).

Recall that the tame symbol ([12, 6]) $(f, g]$ associated to $f$ and $g$ is the line bundle with connection determined (up to isomorphism) by the class $f \cup g$. A "Bloch dilogarithm" ([18]) is (the logarithm of) a horizontal trivializing section, namely a function $L$ on $U \subset X$ satisfying the equation

$$
d L=-\log f d \log g .
$$

Thus $L$ realizes the isomorphism $(f, g] \cong \mathcal{O}_{X}$ over $U \subset X$.

Thus a Bloch dilogarithm will only locally be available, in general. However, when $g=1-f$ then $(1-f, f]$ is globally trivial [6] [18, i.e. $(1-f, f] \cong \mathcal{O}_{X}$. This is the the Steinberg relation satisfied by the Tame symbol. It can be deduced from the following universal case. Set $f=z, X=\mathbb{P}^{1} \backslash\{0,1, \infty\}$, consequently $g=1-z$. Then $L$ is identified with the classical Euler dilogarithm $L i_{2}$, namely

$$
L i_{2}(z)=-\int_{0}^{z} \log (1-t) \frac{d t}{t},
$$


see 12 and 23 for details.

On $\mathbb{P}^{1} \backslash\{0,1, \infty\}$ the classical dilogarithm has a single valued parter, denoted $\mathscr{D}_{2}$, introduced by Bloch and Wigner:

$$
\mathscr{D}_{2}(z)=\arg (1-z) \log |z|+\operatorname{Im} L i_{2}(z) .
$$

$\mathscr{D}_{2}$ is real-analytic on $\mathbb{P}^{1} \backslash\{0,1, \infty\}$ and extends continuously to $\mathbb{P}^{1}$. That it is single-valued can be verified as follows. Choose a branch of the logarithm, say the principal one, to define $L i_{2}$ (and $\mathscr{D}_{2}$ ) on the cut plane $\mathbb{C} \backslash(-\infty, 0] \cup[1, \infty)$. Then one shows that the expression (2.18) is in fact single-valued by analytic continuation along paths based, say, at $1 / 2 \in \mathbb{C}$, and winding around the points 0 and $1 \in \mathbb{C}$. Explicit computations can be found in ref. [7].

It is convenient to introduce

$$
\mathscr{L}_{2}(z)=\sqrt{-1} \mathscr{D}_{2}(z)
$$

so that

$$
d \mathscr{L}_{2}=r_{2}(1-z, z) .
$$

More generally, if $L$ trivializes $(f, g]$ over $U$ in the sense explained above, we can associate a function $\mathscr{L}_{2}(f, g)$ over $U$ such that

$$
d \mathscr{L}_{2}(f, g)=r_{2}(f, g)
$$

via the position

$$
\mathscr{L}_{2}(f, g)=\pi_{1}(\log f) \log |g|+\operatorname{Im} L
$$

\section{Constructions in hermitian holomorphic Deligne cohomology}

In this section we recall the definition of hermitian holomorphic Deligne cohomology. In ref. [10] Brylinski introduced certain complexes $C(l)^{\bullet}$, for a positive integer $l$, in order to compare the Beylinson-Chern classes of a holomorphic vector bundle $E$ with the Cheeger-Chern-Simons classes determined by $(E, \nabla)$, where $\nabla$ is the canonical connection, namely the unique connection compatible with both the holomorphic and hermitian structures. The cohomology groups determined by these complexes are aptly named Hermitian Holomorphic Deligne cohomology groups. For a holomorphic line bundle equipped with the canonical connection, the complex $C(1)^{\bullet}$ encodes the reduction of the structure group from $\mathbb{C}$ to $\mathbb{T}$ afforded by the hermitian fiber metric.

In the following we will need to compute Hermitian Holomorphic Deligne cohomology by means of different-but quasi-isomorphic - sheaf complexes we denote $D(l)_{h}^{\bullet} . h$. These complexes are tailored to a direct description of a metrized line bundle in terms of local representatives of the hermitian fiber metric. Since the two constructions are related by a quasi-isomorphism, the resulting cohomology groups are the same.

\subsection{Hermitian holomorphic Deligne cohomology}

In ref. [10], where Brylinski introduces the complexes:

$$
C(l)^{\bullet}=\operatorname{Cone}\left(\mathbb{Z}(l)_{X} \oplus\left(F^{l} \underline{A}_{X}^{\bullet} \cap \sigma^{2 l} \underline{\varepsilon}_{X}^{\bullet}(l)\right) \longrightarrow \underline{\varepsilon}_{X}^{\bullet}(l)\right)[-1],
$$

where $\sigma^{p}$ denotes the (sharp) truncation in degree $p$, namely for a complex $\underline{F}^{\bullet}, \sigma^{p} \underline{F}^{k}$ is zero for $k<p$ and equal to $\underline{F}^{k}$ for $k \geq p$. In (3.1) we take the cone of the difference between the two inclusions. We have the following:

Definition 3.1 (10 $)$. The hermitian holomorphic Deligne cohomology groups are the hypercohomology groups of the complexes (3.1):

$$
H_{\mathcal{D}_{h . h .}}^{p}(X, l) \stackrel{\text { def }}{=} \mathbf{H}^{p}\left(X, C(l)^{\bullet}\right) .
$$

The complexes (3.1) are expressed as cones, and therefore admit a (standard) Beĭlinson product (2.2). (The wedge product induces cup products on both $\underline{\mathcal{E}}_{X}^{\bullet}(l)$ and $F^{l} \underline{A}_{X}^{\bullet} \cap \sigma^{2 l} \underline{\mathcal{E}}_{X}^{\bullet}(l)$. $)^{3}$ It follows there is a graded commutative product on cohomology:

$$
H_{\mathcal{D}_{h . h .}}^{i}(X, l) \otimes H_{\mathcal{D}_{h . h .}}^{j}(X, k) \stackrel{\cup}{\longrightarrow} H_{\mathcal{D}_{h . h .}}^{i+j}(X, l+k) .
$$

\footnotetext{
${ }^{3}$ It appears signs should be adjusted in the product formula quoted in ref. [10, and that using [2.2 is more appropriate.
} 
Also, from the standard cone exact sequences we get (cf. ref. [10]):

$$
\cdots \longrightarrow H^{2 l-1}(X, \mathbb{R}(l)) \longrightarrow H_{\mathcal{D}_{h . h .}}^{2 l}(X, l) \longrightarrow H^{2 l}(X, \mathbb{Z}(l)) \oplus A^{(l, l)}(X)_{\mathbb{R}(l)} \longrightarrow H^{2 l}(X, \mathbb{R}(l)) \longrightarrow \cdots
$$

where $A^{(l, l)}(X)_{\mathbb{R}(l)}$ denotes the space of smooth $\mathbb{R}(l)$-valued global $(l, l)$-forms on $X$. Thus we see hermitian holormorphic classes are $\mathbb{Z}(l)$-valued classes represented by (necessarily) closed $\mathbb{R}(l)$-valued $2 l$-forms of pure type $(l, l)$. For a line bundle this corresponds to a structure group reduction from $\mathcal{O}_{X}$ to $\underline{\varepsilon}_{X}^{0}$, namely $\mathbb{T}$-valued sections, at the same time controlling the Hodge type of the resulting class, cf. ref. [10]

Later (cf. sec. 3.2) we will want to work with the hermitian structure on a holomorphic line bundle, together with the imaginary $(1,1)$-form built from the canonical connection, directly in the holomorphic frame. To carry this out in general for $(l, l)$ classes, we introduce the complex

$$
D(l)_{h . h .}^{\bullet}=\operatorname{Cone}\left(\mathbb{Z}(l)_{\mathcal{D}}^{\bullet} \oplus\left(F^{l} \underline{A}_{X}^{\bullet} \cap \sigma^{2 l} \underline{\underline{\varepsilon}}_{X}^{\bullet}(l)\right) \longrightarrow \widetilde{\mathbb{R}(l)}\right)_{\mathcal{D}}^{\bullet}[-1] .
$$

The map $\left.\mathbb{Z}(l)_{\mathcal{D}}^{\bullet} \rightarrow \widetilde{\mathbb{R}(l)}\right)_{\mathcal{D}}^{\bullet}$ is the composite of the obvious map $\mathbb{Z}(l)_{\mathcal{D}}^{\bullet} \rightarrow \mathbb{R}(l)_{\mathcal{D}}^{\bullet}$ with the quasi-isomorphism $\rho_{l}$ defined by (2.11). We will simply denote it by $\rho_{l}$ in the sequel, suppressing the first morphism in the notation. The $\operatorname{map}\left(F^{l} \underline{A}_{X}^{\bullet} \cap \sigma^{2 l} \underline{\mathcal{E}}_{X}^{\bullet}(l)\right) \rightarrow \mathbb{R}(l)_{\mathcal{D}}^{\bullet}=\operatorname{Cone}\left(F^{l} \underline{A}_{X}^{\bullet} \rightarrow \underline{\varepsilon}_{X}^{\bullet}(l-1)\right)[-1]$ is induced by the inclusion of $\left(F^{l} \underline{A}_{X}^{\bullet} \cap \sigma^{2 l} \underline{\varepsilon}_{X}^{\bullet}(l)\right)$ into $F^{l} \underline{A}_{X}^{\bullet}$. In (3.5) we take the cone of the difference between these two maps. The complex (3.5) offers another model for Hermitian holomorphic Deligne cohomology. Indeed we have:

Lemma 3.2. The complexes $C(l)^{\bullet}$ and $D(l)_{h . h}^{\bullet}$ are quasi-isomorphic.

Proof. By elementary manipulation of cones

$$
\left.D(l)_{h . h .}^{\bullet}=\operatorname{Cone}\left(F^{l} \underline{A}_{X}^{\bullet} \cap \sigma^{2 l} \underline{\mathcal{E}}_{X}^{\bullet}(l) \rightarrow \operatorname{Cone}\left(\mathbb{Z}(l)_{\mathcal{D}}^{\bullet} \rightarrow \widetilde{\mathbb{R}(l)}\right)_{\mathcal{D}}^{\bullet}\right)\right)[-1]
$$

and clearly: $\operatorname{Cone}\left(\mathbb{Z}(l)_{\mathcal{D}}^{\bullet} \rightarrow \widetilde{\mathbb{R}(l)_{\mathcal{D}}^{\bullet}} \stackrel{\simeq}{\rightarrow} \operatorname{Cone}\left(\mathbb{Z}(l)_{X} \rightarrow \mathbb{R}(l)_{X}\right) \stackrel{\simeq}{\rightarrow} \operatorname{Cone}\left(\mathbb{Z}(l)_{X} \rightarrow \underline{\varepsilon}_{X}^{\bullet}(l)\right)\right.$, where all arrows are quasi-isomorphisms. Thus

$$
\begin{aligned}
D(l)_{h . h .}^{\bullet} & \stackrel{\simeq}{\longrightarrow} \operatorname{Cone}\left(F^{l} \underline{A}_{X}^{\bullet} \cap \sigma^{2 l} \underline{\varepsilon}_{X}^{\bullet}(l) \rightarrow \operatorname{Cone}\left(\mathbb{Z}(l)_{X} \rightarrow \underline{\varepsilon}_{X}^{\bullet}(l)\right)\right)[-1] \\
& =\operatorname{Cone}\left(\mathbb{Z}(l)_{X} \oplus\left(F^{l} \underline{A}_{X}^{\bullet} \cap \sigma^{2 l} \underline{\varepsilon}_{X}^{\bullet}(l)\right) \rightarrow \underline{\varepsilon}_{X}^{\bullet}(l)\right)[-1] \\
& \equiv C(l)
\end{aligned}
$$

as wanted.

It follows from Lemma 3.2 that $\mathbf{H}^{p}\left(X, D(l)_{h . h .}^{\bullet}\right) \cong H_{\mathcal{D}_{h . h .}}^{p}(X, l)$, so we can use either complex to compute the Hermitian-holomorphic Deligne cohomology groups.

Again from the cone exact sequence applied to (3.5), we see the groups $H_{\mathcal{D}_{h . h .}}(X, l)$ also satisfy the exact sequence

$$
\cdots \longrightarrow H_{\mathcal{D}}^{2 l-1}(X, \mathbb{R}(l)) \longrightarrow H_{\mathcal{D}_{h . h .}}^{2 l}(X, l) \longrightarrow H_{\mathcal{D}}^{2 l}(X, \mathbb{Z}(l)) \oplus A^{(l, l)}(X)_{\mathbb{R}(l)} \longrightarrow H_{\mathcal{D}}^{2 l}(X, \mathbb{R}(l)) \longrightarrow \cdots
$$

which we can rewrite as

$$
\cdots \longrightarrow H_{\mathcal{D}}^{2 l-1}(X, \mathbb{R}(l)) \longrightarrow H_{\mathcal{D}_{h . h .}^{2 l}}(X, l) \longrightarrow H_{\mathcal{D}}^{2 l}(X, \mathbb{Z}(l)) \oplus A^{(l, l)}(X)_{\mathbb{R}(l)} \longrightarrow H^{2 l}(X, \mathbb{R}(l)) \cap H^{l, l}(X) \longrightarrow \cdots
$$

Thus the elements of $H_{\mathcal{D}_{h . h}}^{2 l}(X, l)$ map onto those $(l, l)$-forms representing the Hodge classes corresponding to $H_{\mathcal{D}}^{2 l}(X, \mathbb{Z}(l))$.

The complexes $\left.\mathbb{Z}(l)_{\mathcal{D}}^{\bullet}, \widetilde{\mathbb{R}(l)}\right)_{\mathcal{D}}^{\bullet}$, and $F^{l} \underline{A}_{X}^{\bullet} \cap \sigma^{2 l} \underline{\varepsilon}_{X}^{\bullet}(l)$ appearing in the cone (3.5), all have cup products. It follows that we have the Berlinson family of products

$$
D(l)_{h . h .}^{\bullet} \otimes D(k)_{h . h .}^{\bullet} \stackrel{\cup_{\alpha}}{\longrightarrow} D(l+k)_{h . h .}^{\bullet} .
$$

Remark 3.3. The product $\cup_{\alpha}$ in eq. (3.7) should be intended in the modified version provided by (2.5), since the complexes $\mathbb{Z}(l)_{\mathcal{D}}^{\bullet}$ and $\widehat{\mathbb{R}(l)} \dot{\mathcal{D}}^{\bullet}$ have product structures that are compatible with the map $\rho_{l}$ only up to homotopy (given by formula (2.14) $)$. Moreover, these complexes have product structures that are themselves graded commutative up to homotopy: that the product (3.7) is graded commutative up to homotopy follows from Proposition A.4 in the appendix. 


\subsection{Hermitian holomorphic line bundles}

A hermitian holomorphic line bundle or, equivalently, a metrized line bundle, cf. [25, is a holomorphic line bundle $L$ over $X$ together with a hermitian fiber metric $\rho: L \rightarrow \mathbb{R}_{\geq 0}$. As a rule, we will not distinguish $L$ and its sheaf of holomorphic sections. We will also use the alternate notation $|s|_{\rho}$ to denote the length of a local section $s$ of $L$ with respect to $\rho$. Metrized line bundles can be tensor multiplied and an inverse is defined, see. op. cit. An isomorphism of metrized line bundles $(L, \rho)$ and $\left(L^{\prime}, \rho^{\prime}\right)$ is defined in the obvious way, namely it is a map $\phi: L \rightarrow L^{\prime}$ such that $|s|_{\rho}=|\phi(s)|_{\rho^{\prime}}$ for some local section $s$ of $L$. We denote by $\widehat{\operatorname{Pic}(X)}$ the group of isomorphism classes of metrized line bundles.

If $L$ is trivialized over a Čech cover $\mathcal{U}_{X}=\left\{U_{i}\right\}_{i \in I}$ by sections $s_{i}$, then as usual we obtain the cocycle of transition functions $g_{i j} \in \mathcal{O}_{X}^{\times}\left(U_{i} \cap U_{j}\right)$ by writing $s_{j}=s_{i} g_{i j}$. Then if $(L, \rho)$ is a metrized line bundle, we can define the positive function $\rho_{i}=\left|s_{i}\right|_{\rho}^{2}$, namely the local representative of the hermitian structure with respect to the given trivialization. It follows that the various local representatives satisfy

$$
\rho_{j}=\rho_{i}\left|g_{i j}\right|^{2} .
$$

Let us work out the local version of the isomorphism introduced above. Let $s_{i}^{\prime}$ be a local section of $L^{\prime}$ over $U_{i}$. Introduce analogous (primed) quantities for $L^{\prime}$ as we just did for $L$. Given the isomorphism $\phi: L \rightarrow L^{\prime}$ we have $\phi\left(s_{i}\right)=s_{i}^{\prime} f_{i}$, for some $f_{i} \in \mathcal{O}_{X}^{\times}\left(U_{i}\right)$. Then we find $f_{i} g_{i j}=g_{i j}^{\prime} f_{j}$ and $\rho_{i}=\rho_{i}^{\prime}\left|f_{i}\right|^{2}$.

Still working with respect to the chosen cover $\mathfrak{U}_{X}$, a connection compatible with the holomorphic structure is the datum of a collection of $(1,0)$-forms $\xi_{i} \in \underline{A}_{X}^{1,0}\left(U_{i}\right)$ satisfying

$$
\xi_{j}-\xi_{i}=d \log g_{i j} .
$$

Note for future reference that $\underline{A}_{X}^{1,0}=F^{1} \underline{A}_{X}^{1}$. The connection is compatible with the hermitian metric if

$$
\pi_{0}\left(\xi_{i}\right)=\frac{1}{2} d \log \rho_{i}
$$

Using $d=\partial+\bar{\partial}$ and decomposition with respect to $(p, q)$-types, we find the familiar relation

$$
\xi_{i}=\partial \log \rho_{i}
$$

for the unique connection compatible with both the complex and hermitian structures 22 .

The global 2-form

$$
c_{1}(\rho)=\bar{\partial} \partial \log \rho_{i}
$$

represents the first Chern class of $L$ in $H^{2}(X, \mathbb{R}(1))$. Actually, the class of $c_{1}(\rho)$ is a pure Hodge class in $H^{1,1}(X)$ and, according to the examples, it is the image of the first Chern class of $L$ under the map $H_{\mathcal{D}}^{2}(X, \mathbb{Z}(1)) \rightarrow H_{\mathcal{D}}^{2}(X, \mathbb{R}(1))$ induced by $\mathbb{Z}(1) \rightarrow \mathbb{R}(1)$. Observe that $c_{1}(\rho)=c_{1}\left(\rho^{\prime}\right)$ under the isomorphism considered above.

The following proposition can be found, for example, in refs. 99 10. (It apparently was observed first by Deligne, cf. [17.) The proof is based on writing out an explicit cocycle in a Cech resolution of $C(1)^{\bullet}$ or $D(1)_{h . h}^{\bullet}$. This calculation will be needed later on with the complex $D(1)_{h . h}^{\bullet}$, so we provide a proof here.

\section{Proposition 3.4.}

$$
\widehat{\operatorname{Pic}(X)} \cong H_{\mathcal{D}_{h . h .}}^{2}(X, 1)
$$

Proof. Recall that $D(1)_{h . h}^{\bullet}$ is the cone of the map $\rho_{1}-\jmath$, where $\left.\rho_{1}: \mathbb{Z}(1)_{\mathcal{D}}^{\bullet} \rightarrow \widetilde{\mathbb{R}(1)}\right)_{\mathcal{D}}^{\bullet}$, and $\jmath: F^{1} \underline{A}_{X}^{\bullet} \cap \sigma^{2} \underline{\mathcal{E}}_{X}^{\bullet}(1) \rightarrow$ $\mathbb{R}(1)_{\mathcal{D}}^{\bullet}$. By unraveling the structure of all the cones involved we have:

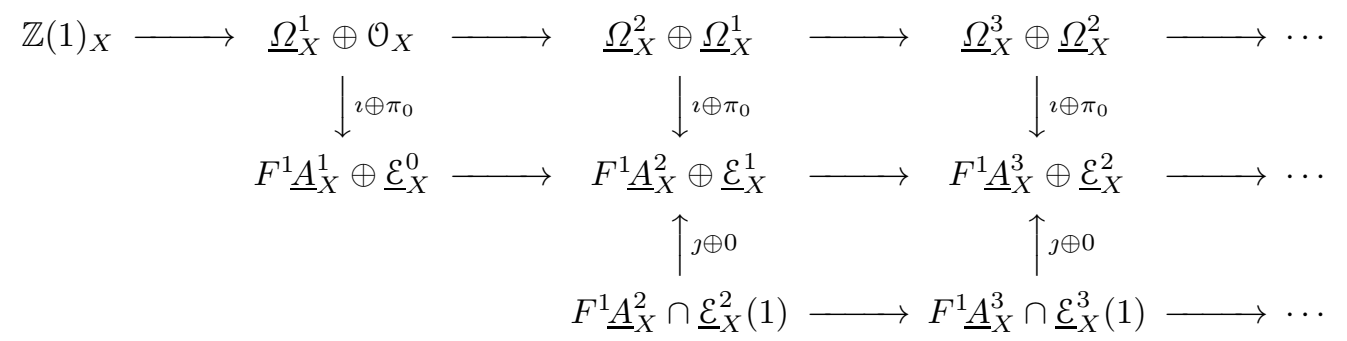


With respect to this diagram, an element of total degree 2 can be written in the form:

\begin{tabular}{c|c|c}
$c_{i j k}$ & $-d \log g_{i j} \oplus \log g_{i j}$ & 0 \\
\hline 0 & $\xi_{i} \oplus \sigma_{i}$ & $\mathrm{X}$ \\
\hline 0 & 0 & $\eta_{i}$
\end{tabular}

for $\xi_{i} \in F^{1} \underline{A}_{X}^{1}\left(U_{i}\right), \sigma_{i} \in \underline{\varepsilon}_{X}^{0}\left(U_{i}\right)$ and $\eta_{i} \in\left(F^{1} \underline{A}_{X}^{2} \cap \underline{\varepsilon}_{X}^{2}(1)\right)\left(U_{i}\right)$. To make sense out of (3.13), note that each entry is an element of the object in the corresponding position in the left $3 \times 3$ part of the previous diagram. Then since the total degree is 2 , the degree of each element in the complex $D(1)_{h . h}^{\bullet}$. is 2 minus the Cech degree as found in (3.13). The top line is the class of $L$ in $\mathbb{Z}(1)_{\mathcal{D}}^{2}$. Finally, the entry marked $\mathbf{X}$ means there is no applicable element-it would have degree 3.

A totally routine calculation shows that (3.13) is a degree 2 cocycle if and only if relations (3.9), (3.10) and $\eta_{i}=d \xi_{i}=2 \bar{\partial} \partial \sigma_{i}$ are satisfied (with $\left.\sigma_{i}=\frac{1}{2} \log \rho_{i}\right)$. Thus $\eta_{i}=\left.c_{1}(\rho)\right|_{U_{i}}$ and all the relations defining a metrized line bundle with its canonical connection are satisfied. The verification that adding an appropriate coboundary to the cocycle leads to an isomorphic metrized bundle in the sense explained above is also routine. Finally, the correspondence between component-wise addition of cocycles modulo coboundaries and the group operation in $\widehat{\operatorname{Pic}(X)}$ is again a direct verification.

From the proof of Proposition 3.4 we have that the cocycle representing $(L, \rho)$ in the Cech resolution of $D(1)_{h . h}^{\bullet}$ has the expression:

\begin{tabular}{c|c|c}
$c_{i j k}$ & $-d \log g_{i j} \oplus \log g_{i j}$ & 0 \\
\hline 0 & $\partial \log \rho_{i} \oplus \frac{1}{2} \log \rho_{i}$ & $\mathbf{X}$ \\
\hline 0 & 0 & $\bar{\partial} \partial \log \rho_{i}$
\end{tabular}

This cocycle is expressed purely in terms of holomorphic and metric data (the local expression for the fiber metric $\rho)$, and it also explicitly encodes the canonical connection and its curvature.

By way of comparison, a degree two cocycle in the Čech resolution of $C(1)^{\bullet}$ with respect to the same cover would be given by a triple

$$
\left(\eta_{i} \oplus \sigma_{i}, \log u_{i j}, c_{i j k}\right),
$$

where $\eta_{i} \in \underline{A}_{X}^{(1,1)}\left(U_{i}\right)_{\mathbb{R}(1)}, \sigma_{i} \in \underline{\varepsilon}_{X}^{1}(1)\left(U_{i}\right), \log u_{i j} \in \underline{\varepsilon}_{X}^{0}(1)\left(U_{i j}\right)$ and $c_{i j k} \in \mathbb{Z}(1)$, satisfying the following relations: aside from the standard cocycle condition for the $\mathbb{T}$-valued functions $u_{i j}$, we must have

$$
\begin{gathered}
d \eta_{i}=0 \\
d \sigma_{i}=\eta_{i}, \\
\sigma_{j}-\sigma_{i}=d \log u_{i j} .
\end{gathered}
$$

Given a holomorphic trivialization of $(L, \rho)$ as above, the position

$$
\eta_{i}=\bar{\partial} \partial \log \rho_{i}, \quad \sigma_{i}=\frac{1}{2} d^{c} \log \rho_{i}, \quad u_{i j}=g_{i j} /\left|g_{i j}\right|
$$

satisfies relations (3.15). From here, the canonical connection is recovered. It is also easily seen that another solution of 3.15) is equivalent to the one above. The following is evident:

Lemma 3.5. Relations (3.16) provide an explicit quasi-isomorphism between the Čech resolutions of $D(1)_{h . h .}^{\bullet}$ and $C(1)^{\bullet}$.

On the other hand, in (3.14) the relations defining the canonical connection are already enforced at the level of the complex.

Finally, if $[L, \rho]$ and $\left[L^{\prime}, \rho^{\prime}\right]$ are the classes corresponding to the metrized bundles $(L, \rho)$ and $\left(L^{\prime}, \rho^{\prime}\right)$, then we write $\left[L \otimes L^{\prime}, \rho \rho^{\prime}\right]=[L, \rho]+\left[L^{\prime}, \rho^{\prime}\right]$. 


\subsection{Cup product of hermitian holomorphic line bundles}

If $L$ and $L^{\prime}$ are two line bundles on $X$, their cup product in Deligne cohomology would produce a class in $H_{\mathcal{D}}^{4}(X, \mathbb{Z}(2))$ denoted $\left(L, L^{\prime}\right]$. Calculations were carried out in [9] where a geometric interpretation of $\left(L, L^{\prime}\right]$ as a 2 -gerbe and its connection with the determinant of cohomology (when $X$ is the total space of a family of Riemann surfaces) were put forward. The structure ensuing from the generalization to line bundles equipped with a hermitian metric was further analyzed in ref. 10 by means of hermitian holomorphic Deligne cohomology.

The cup product of two metrized line bundles $(L, \rho)$ and $\left(L^{\prime}, \rho^{\prime}\right)$ in hermitian holomorphic Deligne cohomology produces a class in $H_{\mathcal{D}_{h, h}}^{4}(X, 2)$. Despite the more involved definition of $D(l)_{h . h}^{\bullet}$. as opposed to that of $C(l)^{\bullet}$, it will nonetheless be more advantageous from the perspective of sect. 5 to use the former to calculate the desired class. Thus let us explicitly compute a representative of this class in terms of the expression (3.14) for the class of a metrized line bundle and the modified Beilinson product (2.5) for

$$
D(1)_{h . h .}^{\bullet} \otimes D(1)_{h . h .}^{\bullet} \stackrel{\cup_{\alpha}}{\longrightarrow} D(2)_{h . h .}^{\bullet} .
$$

A diagram for the complex $D(2)_{h . h}^{\bullet}$. analogous to that for $D(1)_{h . h}^{\bullet}$ displayed in the proof of Proposition 3.4 is:

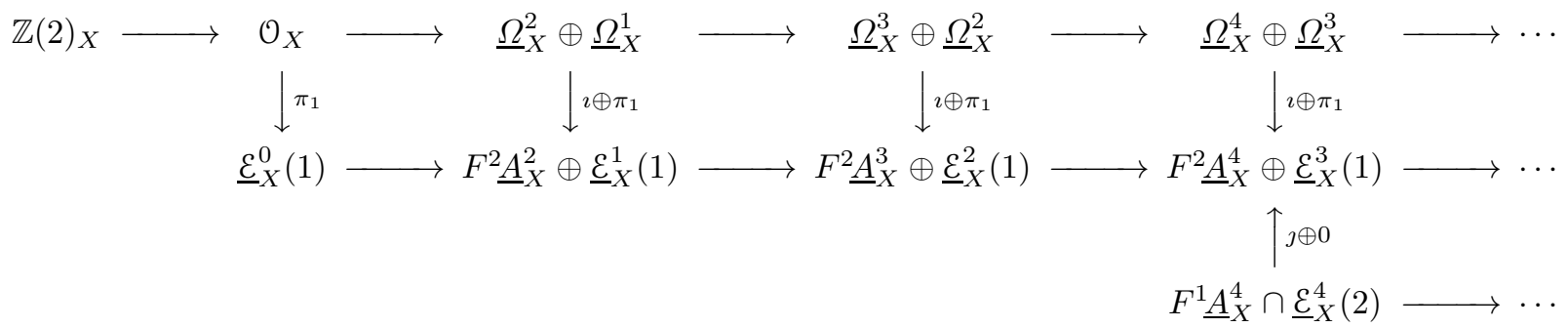

The cup product of two elements of the form (3.14) in the Čech resolution of $D(1)_{h . h .}^{\bullet}$ would result in an analogous $5 \times 3$ table. The degrees (in the cone) start from 0 in the leftmost entry in the top and bottom rows, and from 1 in the center one. The overall signs are determined by those in the Beillinson product (2.5) plus those arising from the Čech resolution as explained in sect. 1.2 Actually, it is visually less cumbersome to display the resulting expression as in (3.18) below, where the corresponding bidegrees are explicitly indicated: the first degree is the overall degree in the cone and the second is the Čech degree. (We have explicitly written only the nonzero terms.)

\begin{tabular}{c|c}
$(0,4)$ & $c_{i j k} c_{k l m}^{\prime}$ \\
\hline$(1,3)$ & $-c_{i j k} \log g_{k l}^{\prime}$ \\
\hline$(2,2)$ & $\left(-d \log g_{i j} \wedge d \log g_{j k}^{\prime} \oplus-\log g_{i j} d \log g_{j k}^{\prime}\right) \oplus-\pi_{1} \log g_{i j} \log \left|g_{j k}^{\prime}\right|$ \\
\hline$(3,1)$ & $\left(-(1-\alpha) d \log g_{i j} \wedge \partial \log \rho_{j}^{\prime}+\alpha \partial \log \rho_{i} \wedge d \log g_{i j}^{\prime}\right) \oplus\left\{(1-\alpha)\left(d^{c} \log \left|g_{i j}\right| \frac{1}{2} \log \rho_{j}^{\prime}-\log \left|g_{i j}\right| \frac{1}{2} d^{c} \log \rho_{j}^{\prime}\right)\right.$ \\
& $\left.+\alpha\left(-\frac{1}{2} d^{c} \log \rho_{i} \log \left|g_{i j}^{\prime}\right|+\frac{1}{2} \log \rho_{i} d^{c} \log \left|g_{i j}^{\prime}\right|\right)\right\}$ \\
\hline$(4,0)$ & $\bar{\partial} \partial \log \rho_{i} \wedge \bar{\partial} \partial \log \rho_{i}^{\prime} \bigoplus\left(\alpha \bar{\partial} \partial \log \rho_{i} \wedge \partial \log \rho_{i}^{\prime}+(1-\alpha) \partial \log \rho_{i} \wedge \bar{\partial} \partial \log \rho_{i}^{\prime}\right)$ \\
& $\oplus\left(\alpha \bar{\partial} \partial \log \rho_{i} \frac{1}{2} \log \rho_{i}^{\prime}+(1-\alpha) \frac{1}{2} \log \rho_{i} \bar{\partial} \partial \log \rho_{i}^{\prime}\right)$
\end{tabular}

\subsection{Integration along the fiber}

To conclude this introduction, let us quickly mention that Hermitian holomorphic Deligne cohomology has an integration along the fiber map. Namely, if $\pi: X \rightarrow S$ is a proper submersion of complex manifolds, it follows from [20] that Deligne cohomology has an integration along the fiber $\int_{\pi}$, hence by [9, 10] there is a map

$$
H_{\mathcal{D}_{h . h .}}^{i}(X, l) \longrightarrow H_{\mathcal{D}_{h . h .}}^{i-2 d}(S, l-d)
$$

where $d$ is the complex dimension of the fiber, and commutative diagrams analogous to [10. Theorem 5.1].

We will be interested in the case of complex relative dimension 1, namely $\pi: X \rightarrow S$ is a holomorphic fibration with compact connected Riemann surfaces as fibers. From (3.19) in degree 4 we have the map: $H_{\mathcal{D}_{h . h .}}^{4}(X, 2) \rightarrow$ $H_{\mathcal{D}_{h . h .}}^{2}(S, 1)$ into the group of complex hermitian line bundles on $S$ (cf. Proposition 3.4).

We refer to refs. 9] 10] for a complete treatment of the map (3.19), in particular for the "trace" map

$$
\mathbf{R}^{\bullet} \pi_{*}\left(D(l)_{h . h .}^{\bullet}\right) \longrightarrow D(l-d)_{h . h .}^{\bullet-2 d},
$$


which induces (3.19) at the level of cohomology. (Clearly any quasi-isomorphic model for Hermitian-holomorphic cohomology will do.) We will limit ourselves to observe the following: if $\mathfrak{U}_{S}$ is a good cover of $S$, and $\mathfrak{U}_{X}$ a good cover of $X$ which refines $\pi^{-1} \mathcal{U}_{S}$, the $i$-th direct image $\mathbf{R}^{i} \pi_{*} \underline{F}^{\bullet}$ of any complex $\underline{F}^{\bullet}$ on $X$ is the sheaf on $S$ associated to the presheaf

$$
V \longmapsto H^{i}\left(\operatorname{Tot} \check{C}^{\bullet}\left(\mathcal{U}_{X} \cap \pi^{-1}(V), \underline{F}^{\bullet}\right)\right)
$$

Then unraveling the cone structure of $D(l)_{h . h . X}^{\bullet^{\prime}}$ (or of $C(l)_{X}^{\bullet}$ ) reduces to computing the direct images of $\mathbb{Z}(l)_{X}$ and $\underline{A}_{X}^{\bullet}$, the de Rham complex. The trace map is then obtained by capping a total cocycle, say in

$$
\bigoplus_{p+q=i} \check{C}^{q}\left(\mathcal{U}_{X} \cap \pi^{-1}(V), \underline{A}_{X}^{p}\right)
$$

with a representative of the fundamental class $[M]$ of the smooth model $M$ of the fiber of $\pi^{-1}(V) \cong V \times M$ (as smooth manifolds). Explicit representatives for $[M]$ for a triangulation subordinated to the nerve of $\mathcal{U}_{X} \cap \pi^{-1}(V)$ are computed in ref. [3]. If $\omega_{i_{0}, \ldots, i_{q}}^{p}$ are the components of a cocycle $\omega_{V}^{i}$ in (3.20), then

$$
\omega_{V}^{i} \cap[M]=\sum \omega_{i_{0}, \ldots, i_{q}}^{p} \cap \Delta_{i_{0}, \ldots, i_{q}}^{2 d-q}
$$

where the $\Delta_{i_{0}, \ldots, i_{q}}^{2 d-q}$ are signed generators of the nerve of dimension $2 d-q$. The exact combinatorics can be found in ref. [3], so we will not further pursue the matter here.

Let us conclude by noticing that for $\pi: X \rightarrow S$ the cup product of $(L, \rho)$ and $\left(L^{\prime}, \rho^{\prime}\right)$ induces a metrized line bundle on $S$. From the preceding discussion, the connection component, for example, will be obtained by following the (two) components $F^{2} \underline{A}_{X}^{\bullet} \cap \underline{\varepsilon}_{X}^{\bullet \geq 4}(2)$ in (3.18). The one in degree $(4,0)$ will be capped with generators $\Delta^{2}$ of dimension 2 (hence integrated over 2-simplices), those in degree $(3,1)$ with generators $\Delta^{1}$ of dimension 1.

From [9, Theorem 6.1] there is a corresponding map $A^{(2,2)}(X)_{\mathbb{R}(2)} \rightarrow A^{(1,1)}(S)_{\mathbb{R}(1)}$. From the explicit cocycles we have computed the corresponding representative in $H^{2}(S, \mathbb{R}(1)) \cap H^{1,1}(S)$ of the first Chern class of the resulting metrized line bundle is

$$
\int_{\pi} c_{1}(\rho) \wedge c_{1}\left(\rho^{\prime}\right)
$$

as in [11, Proposition 6.6.1].

\section{Conformal metrics and the Liouville functional}

Let $X$ be a compact Riemann surface of genus $g \geq 2$. For simplicity we can assume $X$ to be connected, although this is not necessary, and in fact this assumption will be dropped when dealing with Kleinian groups.

Let $\mathscr{C} \mathscr{M}(X)$ be the space of conformal metrics on $X$. Locally on $X$, if $z$ is a local analytic coordinate defined on an open set $U$, any metric $d s^{2}$ can be represented as

$$
d s^{2}=\rho|d z|^{2}
$$

for a positive function $\rho: U \rightarrow \mathbb{R}_{>0}$. According to sec. 3.2 a conformal metric corresponds to considering the metrized line bundle $\left(T_{X}, \rho\right)$, where $T_{X}$ is the holomorphic tangent bundle of $X$. With respect to a cover $\mathfrak{U}_{X}$ of $X$, the conformal factors $\rho_{i}$ and $\rho_{j}$, associated to $U_{i}$ and $U_{j}$ respectively, satisfy the relation

$$
\rho_{j}=\rho_{i}\left|z_{i j}^{\prime}\right|^{2},
$$

where $z_{i j}^{\prime}=d z_{i} / d z_{j}$ and $z_{i}$ is a local coordinate defined over $U_{i}$.

It follows from the uniformization theorem that there exists a unique conformal metric of scalar curvature equal to -1 , the Poincaré metric. Locally on $U_{i} \subset X$, the condition for the metric to have curvature -1 is equivalent to the nonlinear PDE

$$
\frac{\partial^{2}}{\partial z_{i} \partial \bar{z}_{i}} \phi_{i}=\frac{1}{2} \exp \phi_{i}
$$

known as the Liouville equation, for the smooth function $\phi_{i}=\log \rho_{i}$. Observe that equation (4.2) can be written in the form

$$
c_{1}(\rho)=\sqrt{-1} \omega_{\rho},
$$


where we have used the Kähler form associated to the metric:

$$
\left.\omega_{\rho}\right|_{U_{i}}=\frac{\sqrt{-1}}{2} \rho_{i} d z_{i} \wedge d \bar{z}_{i} .
$$

This representation makes it apparent that the Liouville equation is independent of the choice of the coordinate system. On the other hand, a direct verification of this fact is immediate using $\phi_{j}-\phi_{i}=\log \left|z_{i j}^{\prime}\right|^{2}$.

\subsection{Variational problem for conformal metrics}

It is well known that the Liouville equation has a local variational principle in the following sense. Let $D$ be a region in the complex plane. Then equation (4.2) is the Euler-Lagrange equation for the variational problem defined by the action functional

$$
S[\phi]=\frac{\sqrt{-1}}{2} \int_{D}\left(\partial \phi \wedge \bar{\partial} \phi+e^{\phi} d z \wedge d \bar{z}\right)
$$

defined on smooth functions $\phi: D \rightarrow \mathbb{R}$, with the condition that variations $\left.\frac{d}{d \alpha}\left(\phi_{\alpha}\right)\right|_{\alpha=0}$ of $\phi$ be zero on $\partial D$. However, it is easily seen that the functional (4.3) cannot be defined globally on $X$, since, as a consequence of (4.1), the first term under the integral sign would not yield a well-defined 2-form on $X$. (The second one would of course present no problems, it would just give the area $A_{X}(\rho)$ of $X$ with respect to the given metric $\rho|d z|^{2}=e^{\phi}|d z|^{2}$.) Accordingly, it is convenient to write the integrand in (4.3) as

$$
\sqrt{-1} \omega[\phi]+\omega_{\rho}
$$

where we have defined the 2 -form ${ }^{4}$

$$
\omega^{0}[\phi]=\frac{1}{2} \partial \phi \wedge \bar{\partial} \phi
$$

and restrict our considerations to the first term of (4.3) which we denote

$$
\check{S}[\phi]=\sqrt{-1} \int_{D} \omega^{0}[\phi] .
$$

There is by now an established procedure on how to address the problem caused by the fact that (4.4) is not globally defined. In general terms, given the choice of a conformal metric $d s^{2} \in \mathscr{C} \mathscr{M}(X)$ and a cover $\mathfrak{U}_{X}$, one works with the full Cech-de Rham complex $\check{C}^{\bullet}\left(\mathcal{U}_{X}, \underline{\varepsilon}_{X}^{\bullet}(1)\right)$ with respect to $\mathcal{U}_{X}$, rather than with just differential forms. The 2-form (4.4) is then completed to a total degree 2-cocycle - to be denoted $\Omega[\phi]$. This results in a class in $H^{2}(X, \mathbb{R}(1))$ after taking cohomology. (Whether or not there also is a de Rham type theorem will depend on the acyclicity properties of $\mathcal{U}_{X}$.)

This scheme has been previously carried out not quite for covers of $X$ by open sets, but actually for different choices of planar coverings. For the covering associated to a Schottky uniformization of $X$ a generalization of eq. (4.3) was written in ref. 34. More recently, a detailed calculation of the cocycle for the general case of a covering associated to a Kleinian uniformization was carried out in ref. 30 by exploiting the homological methods developed in ref. [2]. Note, however, that from the point of view of ref. [3] these planar coverings are "étale" coordinates on $X$, so the group cohomology constructions required to work with the various kinds of uniformization coverings just follow from specializing the Cech formalism to the coverings at hand.

Finally, the integration in eqs. (4.3) or (4.5) should be replaced by the evaluation of $\Omega[\phi]$ against an appropriate representative $\Sigma$ of the fundamental class of $X$. The "appropriate" form for both $\Sigma$ and the evaluation will be dictated by the chosen cover $\mathcal{U}_{X}$ and the cohomology theory being used. Typically $\Sigma$ will be a cycle in a double complex of $\mathfrak{U}_{X}$-small simplices, where the differentials are the singular one and the one determined by the face maps induced by the cover. Thus, in the case of a Čech cover, it will be the complex determined by a triangulation of $X$ subordinated to the open $\mathcal{U}_{X}$, and in the same way, for a planar cover the singular complex of the planar domain $\mathfrak{U}_{X}$ tensored with an appropriate bar resolution of the group of deck transformations. These issues have been discussed at length in refs. 22, 3, 30, so we will not repeat the discussion here. Whenever we have a cocycle extending (4.4) and a cycle $\Sigma$ representing $X$ we state

\footnotetext{
${ }^{4}$ Note that equation (4.4) defines an imaginary form. The reason for this choice will be apparent later.
} 
Definition 4.1. The Liouville functional (without the area term) is given by the evaluation:

$$
\check{S}[\phi]=-\frac{1}{2 \pi \sqrt{-1}}\langle\Omega[\phi], \Sigma\rangle .
$$

For the complete functional we add the area term

$$
S[\phi]=\check{S}[\phi]+\frac{1}{2 \pi} \int_{X} \omega_{\rho} .
$$

Remark 4.2. $\langle\Omega[\phi], \Sigma\rangle \in \mathbb{R}(1)$, and $\check{S}[\phi]$ (or $S[\phi]$ ) is real. Division by $2 \pi \sqrt{-1}$ is conventional, but note that $\mathbb{R}(1) \stackrel{\simeq}{\rightarrow} \mathbb{R}_{+}$via $\exp (\cdot / 2 \pi \sqrt{-1})$. In the sequel it will be more convenient to work with imaginary classes. (See also section [5)

In next two subsections we examine these constructions in some detail. For definiteness, we initially make use of an ordinary open cover. First, we recall the direct construction of a cocycle generalizing (4.4), and we show that this way eq. (4.2) indeed is the resulting extremum condition. Then we emphasize the role played by Deligne cohomology and the tame symbol. These aspects will become important when introducing a Kleinian uniformization later in the paper, when we discuss connections with the dilogarithm function. In a later section we shall tackle the question of its geometrical significance by making full use of the hermitian-holomorphic version of Deligne cohomology presented in section [3] and we show that the cocycle constructed following refs. [34, 30] corresponds to the square of $\left(T_{X}, \rho\right)$ in hermitian holomorphic Deligne cohomology.

\subsection{Direct construction of the Liouville cocycle}

\subsubsection{Initial setup}

Let $X$ a compact Riemann surface of genus greater than 2. We shall not include the area term in our explicit calculations, therefore it makes sense to extend our considerations to a general metrized line bundle $(L, \rho)$. Of course, whenever referring to a conformal metric or to the variational problem for the Liouville equation, it will be be assumed that $L=T_{X}$ and that $g_{i j}=z_{i j}^{\prime}$. Upon choosing a cover $\mathcal{U}_{X}$, which for now we assume to be a Čech cover by open sets, the pair $(L, \rho)$ is described in terms of the data expounded in section 3.2 Our starting point will be the 0-cochain

$$
\omega_{i}^{0}\left[\log \rho_{i}\right]=\frac{1}{2} \partial \log \rho_{i} \wedge \bar{\partial} \log \rho_{i}=-\frac{1}{2} d \log \rho_{i} \wedge \frac{1}{2} d^{c} \log \rho_{i}
$$

with values in $\underline{\varepsilon}_{X}^{2}(1)\left(U_{i}\right)$.

Remark 4.3. A generalization for eq. (4.8) would be to consider a pair of metrized line bundles $(L, \rho)$ and $\left(L^{\prime}, \rho^{\prime}\right)$, and then the analog of (4.8) would be

$$
\omega_{i}^{0}\left[\log \rho_{i}, \log \rho_{i}^{\prime}\right]=\frac{1}{2}\left(-\frac{1}{2} d \log \rho_{i} \wedge \frac{1}{2} d^{c} \log \rho_{i}^{\prime}+\frac{1}{2} d^{c} \log \rho_{i} \wedge \frac{1}{2} d \log \rho_{i}^{\prime}\right)
$$

Note, however, that the expressions are quadratic. Moreover, $L \otimes L^{\prime}$ has metric $\rho \rho^{\prime}$, so that there is a natural "polarization identity"

$$
\omega\left[\log \rho, \log \rho^{\prime}\right]=\frac{1}{4} \omega\left[\log \rho \rho^{\prime}\right]-\frac{1}{4} \omega\left[\log \frac{\rho}{\rho^{\prime}}\right]
$$

where we have omitted the indexes for simplicity of notation. We shall comment later on the significance of eq. (4.10).

\subsubsection{Computation}

Let us extend (4.8) to a degree 2 cocycle in the total simple complex associated to the double complex $\check{C}^{\bullet}\left(\mathcal{U}_{X}, \underline{\varepsilon}_{X}^{\bullet}(1)\right)$ of Cech cochains with values in the de Rham complex of imaginary smooth forms. This is accomplished in the usual fashion (see e.g. [8]) by finding a 1-cochain of 1-forms $\omega_{i j}^{1}[\log \rho]$ on $U_{i j}$ and a 2-cochain of 0 -forms $\omega_{i j k}^{2}[\log \rho]$ on $U_{i j k}$ such that the relations

$$
\check{\delta} \omega^{0}=-d \omega^{1}, \quad \check{\delta} \omega^{1}=d \omega^{2}, \quad \check{\delta} \omega^{2}=0
$$


are satisfied. Of course, the remaining one, namely $d \omega=0$ is automatically satisfied for dimensional reasons. It turns out that to a great extent these relations are explicitly computable without further assumptions, such as that the cover $\mathcal{U}_{X}$ be good. The needed calculations are fairly standard, and they are presented in great detail in ref. [30, so we shall be brief. (The observation in [25] that on a Riemann surface for two smooth functions $f$ and $g$ one has $d f \wedge d^{c} g=d g \wedge d^{c} f$ is useful in carrying out the calculations.) The first two steps are as follows.

First, one has:

$$
\begin{gathered}
\omega_{j}^{0}[\rho]-\omega_{i}^{0}[\rho]=-d \omega_{i j}^{1}[\rho] \\
\omega_{i j}^{1}[\rho]=\frac{1}{2} \log \rho_{i} d^{c} \log \left|g_{i j}\right|+d^{c} \log \left|g_{i j}\right| \frac{1}{2} \log \rho_{j}
\end{gathered}
$$

The next step yields:

$$
\begin{aligned}
\check{\delta}\left(\omega^{1}[\log \rho]\right)_{i j k} & =\omega_{j k}^{1}[\rho]-\omega_{i k}^{1}[\rho]+\omega_{i j}^{1}[\rho] \\
& =\log \left|g_{i j}\right| d^{c} \log \left|g_{j k}\right|-d^{c} \log \left|g_{i j}\right| \log \left|g_{j k}\right|
\end{aligned}
$$

and notice that $d^{c} \log \left|g_{i j}\right|=\pi_{1} d \log g_{i j}$, and $\pi_{p+q-1}(a \wedge b)=\pi_{p}(a) \wedge \pi_{q-1}(b)+\pi_{p-1}(a) \wedge \pi_{q}(b)$, so we have

$$
\check{\delta}\left(\omega^{1}[\log \rho]\right)_{i j k}=\pi_{1}\left(\log g_{i j} d \log g_{j k}\right)-d\left(\pi_{1}\left(\log g_{i j}\right) \log \left|g_{j k}\right|\right) \text {. }
$$

Observe that now the problem of continuing the descent becomes independent of the chosen metric $\rho$. The most direct way of proceeding is the following. If we assume the cover $\mathcal{U}_{X}$ to be acyclic for the de Rham complex $\underline{\mathcal{E}}_{X}^{\bullet}(1)$, then there exists $\omega_{i j k}^{2} \in \underline{\mathcal{E}}_{X}^{0}(1)\left(U_{i j k}\right)$ such that $d \omega_{i j k}^{2}=\check{\delta}\left(\omega^{1}[\log \rho]\right)_{i j k}$. Furthermore, consistency on a quadruple intersection requires that $\check{\delta} \omega^{2}$ be a 3 -cocycle with values in $\mathbb{R}(1)_{X}$. Since $H^{3}(X, \mathbb{R}(1))=0$, this cocycle must be a coboundary, therefore, up to readjusting the constants, there exists a choice of $\omega_{i j k}^{2}$ such that $\check{\delta} \omega^{2}=0$, and furthermore, $\omega_{i j k}^{2}$ does not depend on the metric structure.

\subsubsection{Solution to the variational problem}

The previous preliminary calculation is sufficient from the point of view of finding the extrema. To this effect, we set $L=T_{X}$, for $\rho$ a conformal metric in $\mathscr{C} \mathscr{M}(X)$. Notice that the space of conformal metrics on $X$ is affine over $C^{\infty}(X, \mathbb{R}) \equiv \mathcal{E}^{0}(X)$ : if $d s^{2}$ and $d s^{\prime 2}$ are two conformal metrics with local expressions $\rho_{i}\left|d z_{i}\right|^{2}$ and $\rho_{i}^{\prime}\left|d z_{i}\right|^{2}$ respectively, then there exists $\sigma \in C^{\infty}(X, \mathbb{R})$ such that $\log \rho_{i}^{\prime}=\left.\sigma\right|_{U_{i}}+\log \rho_{i}$. The change from $\Omega[\log \rho]$ to $\Omega[\log \rho+\sigma]$ can be exactly computed thanks to the fact that the last step in the determination of $\Omega[\log \rho]$ is independent of $\rho$ and the quadratic character of (4.4). Indeed we have:

\section{Proposition 4.4.}

$$
S[\log \rho+\sigma]-S[\log \rho]=\frac{1}{2 \pi \sqrt{-1}} \int_{X}\left(\frac{1}{2} \partial \sigma \wedge \bar{\partial} \sigma+\sigma c_{1}(\rho)-\sqrt{-1}\left(e^{\sigma}-1\right) \omega_{\rho}\right)
$$

Proof. The change in $\omega_{i}^{0}[\log \rho]$ is computed as

$$
\omega_{i}^{0}[\log \rho+\sigma]-\omega_{i}^{0}[\log \rho]=-\frac{1}{2} d \sigma_{i} \wedge \frac{1}{2} d^{c} \sigma_{i}+\sigma_{i} \frac{1}{2} d d^{c} \log \rho_{i}-\frac{1}{2} d\left(\sigma_{i} d^{c} \log \rho_{i}\right)
$$

where we set $\left.\sigma_{i} \equiv \sigma\right|_{U_{i}}$. Note that the first two terms on the right hand side are globally well-defined 2-forms. On the other hand

$$
\omega_{i j}^{1}[\log \rho+\sigma]-\omega_{i j}^{1}[\log \rho]=\sigma_{i} d^{c} \log \left|g_{i j}\right| .
$$

Letting $\chi_{i}=\sigma_{i} d^{c} \log \rho_{i}$, we see that

$$
\Omega[\log \rho+\sigma]-\Omega[\log \rho]=-\frac{1}{2} d \sigma \wedge \frac{1}{2} d^{c} \sigma+\sigma \frac{1}{2} d d^{c} \log \rho-D \chi,
$$

and taking the area terms into account, establishes the formula.

As a consequence, we obtain

Corollary 4.5. The Liouville equation (4.2) is the Euler-Lagrange equation for the Liouville functional (4.7) introduced in definition 4.1. The critical point is non-degenerate. 
Proof. Replacing $\sigma$ with $t \sigma, t \in \mathbb{R}$, in the previous proposition we find the infinitesimal change in $\Omega[\log \rho]$ to be

$$
\left.\frac{d}{d t}\right|_{t=0} S[\log \rho+t \sigma]=-\frac{1}{2 \pi \sqrt{-1}} \int_{X} \sigma\left(c_{1}(\rho)-\sqrt{-1} \omega_{\rho}\right),
$$

and it follows that $S[\log \rho]$ has an extremum if and only if the Liouville equation is satisfied. Non degeneracy follows from the quadratic part in the exact change formula in proposition 4.4

Remark 4.6. The fact that the change in the cocycle is given by a pure 2-form term up to total coboundary can also be analyzed in terms of gluing properties of variational bicomplexes, cf. Theorem 1.2 in ref. 1 and the proof of Theorem 1 in ref. 3]. From this perspective, Corollary 4.5] is a direct consequence of the affine structure of the space $\mathscr{C} \mathscr{M}(X)$ of conformal metrics.

\subsection{A cup product}

Formula (4.12) can be handled in a more geometric fashion as follows. From sec. 2.4 we can rewrite (4.12) as

$$
\check{\delta}\left(\omega^{1}[\log \rho]\right)_{i j k}=-r_{2}\left(g_{i j}, g_{j k}\right),
$$

and we have the collection of tame symbols $\left(g_{i j}, g_{j k}\right]$ associated any triple intersection $U_{i j k}$ in the cover $\mathcal{U}_{X}, 9$. These symbols glue to form a global symbol $(L, L]$. As a cohomology class on a curve $X$, however, $(L, L]$ will be zero (that is, there is a global object in the associated 2-stack, cf. [10]), so that it will be possible to choose local functions $L_{i j k}$ such that

$$
d L_{i j k}=-\log g_{i j} d \log g_{j k},
$$

as explained in detail in [3]. Note that we still need the cover $\mathcal{U}_{X}$ to be fine enough. Moreover, cf. loc. cit., the collection $L_{i j k}$ can be chosen in a way that

$$
\check{\delta} L_{i j k l}=-c_{i j k} \log g_{k l}+n_{i j k l},
$$

where $n_{i j k l} \in \mathbb{Z}(2)$. Therefore from (2.19), (4.12) and (4.13), we can set

$$
\omega_{i j k}^{2}=-\left(\pi_{1}\left(\log g_{i j}\right) \log \left|g_{j k}\right|\right)-\pi_{1} L_{i j k},
$$

namely according to sec. 2.4 we have set $\omega_{i j k}^{2}=-\mathscr{L}_{2}\left(g_{i j}, g_{j k}\right)$. Now, the last compatibility condition is satisfied, indeed we have:

$$
\begin{aligned}
\check{\delta} \omega_{i j k l}^{2} & =-c_{i j k} \log \left|g_{k l}\right|-\pi_{1}\left(\check{\delta} L_{i j k l}\right) \\
& =-c_{i j k} \log \left|g_{k l}\right|+\pi_{1}\left(c_{i j k} \log g_{k l}\right) \\
& =0 .
\end{aligned}
$$

As a result, we obtain a 2-cocycle in the the total complex associated to $\check{C}^{\bullet}\left(\mathcal{U}_{X}, \underline{\varepsilon}_{X}^{\bullet}(1)\right)$ as before, with a more geometric interpretation of $\omega_{i j k}^{2}$ in terms of the trivialization of the symbol $(L, L]$. Moreover, notice that if $X$ is a curve then $\left.\underline{\mathcal{E}}_{X}^{\bullet}(1)[1] \stackrel{\simeq}{\longrightarrow} \widetilde{\mathbb{R}(2)}\right)_{\mathcal{D}}^{\bullet}$ thus we may interpret the class so determined by $\Omega[\log \rho]=\omega^{0}[\log \rho]+\omega^{1}[\log \rho]+\omega^{2}$ as a (degree 3$)$ class in $H_{\mathcal{D}}^{3}(X, \mathbb{R}(2))$.

\subsection{Two line bundles}

For a pair $(L, \rho),\left(L^{\prime}, \rho^{\prime}\right)$ of metrized line bundles we can complete (4.9) to a cocycle $\Omega\left[\log \rho, \log \rho^{\prime}\right]$ via an analogous procedure to the one presented in sects. 4.2 and 4.3 The relevant calculations being entirely similar, we limit ourselves to quoting the relevant expressions. Starting from (4.9), which we rewrite in the form

$$
\omega_{i}^{0}\left[\log \rho_{i}, \log \rho_{i}^{\prime}\right]=\frac{1}{2} d^{c} \log \rho_{i} \wedge \frac{1}{2} d \log \rho_{i}^{\prime},
$$

the corresponding expression for the degree $(1,1)$ term is:

$$
\omega_{i j}^{1}\left[\log \rho, \log \rho^{\prime}\right]=\frac{1}{2} \log \rho_{i} d^{c} \log \left|g_{i j}^{\prime}\right|+\frac{1}{2} d^{c} \log \left|g_{i j}\right| \log \rho_{j}^{\prime} .
$$


Computing the Čech coboundary we find:

$$
\begin{aligned}
\left(\check{\delta} \omega^{1}\left[\log \rho, \log \rho^{\prime}\right]\right)_{i j k} & =\log \left|g_{i j}\right| d^{c} \log \left|g_{j k}^{\prime}\right|-d^{c} \log \left|g_{i j}\right| \log \left|g_{j k}^{\prime}\right| \\
& =-r_{2}\left(g_{i j}, g_{j k}^{\prime}\right),
\end{aligned}
$$

from which $\omega_{i j k}^{2}$ (now independent of $\rho$ and $\rho^{\prime}$ ) can be obtained as $-\mathscr{L}_{2}\left(g_{i j}, g_{j k}^{\prime}\right)$ by looking at a collection $L_{i j k}$ such that

$$
d L_{i j k}=-\log g_{i j} d \log g_{j k}^{\prime},
$$

from the triviality of the symbol $\left(L, L^{\prime}\right]$.

\subsection{Additional remarks on the Liouville functional and the Bloch-Wigner diloga- rithm}

We wish to compare the previous constructions to those of ref. [30]. To this end we need to specifically consider the case of a cover of $X$ provided by a Kleinian uniformization. (The reader should consult loc. cit. for reference and complete details.) The comparison offers a better perspective on the absence of cohomological obstructions in the calculations of ref. [30, and on the relations with three-dimensional hyperbolic geometry.

Let $\Gamma$ be a purely loxodromic second kind Kleinian group satisfying all the conventions spelled out in Appendix $B$ to which we refer for the notation. Let $U_{\Gamma} \subset \mathbb{P}^{1}$ be the region of discontinuity, and $X=U_{\Gamma} / \Gamma$ the resulting (possibly disconnected) Riemann surface.

A conformal metric $\rho$ on $X=X_{1} \sqcup \cdots \sqcup X_{n}$ appears as an automorphic function on $U_{\Gamma}$ :

$$
\frac{1}{2} \log \rho-\frac{1}{2} \log \rho \circ \gamma=\log \left|\gamma^{\prime}\right|, \quad \gamma \in \Gamma .
$$

Eq. (4.18) is the direct translation of (3.8) following the principles of 3]. Accordingly, the first two terms of the Liouville cocycle computed by applying the procedure explained in sect. 4 - are:

$$
\begin{gathered}
\omega^{0}[\log \rho]=-\frac{1}{2} d \log \rho \wedge \frac{1}{2} d^{c} \log \rho \\
\omega_{\gamma}^{1}[\log \rho]=\left(\frac{1}{2} \log \rho+\frac{1}{2} \log \rho \circ \gamma\right) d^{c} \log \left|\gamma^{\prime}\right|
\end{gathered}
$$

and computing the coboundary of (4.20) according to the prescription in Appendix B yields

$$
\check{\delta}\left(\omega^{1}[\log \rho]\right)_{\gamma_{1}, \gamma_{2}}=-r_{2}\left(\left(\gamma_{1} \gamma_{2}\right)^{\prime}, \gamma_{2}^{\prime}\right),
$$

where $r_{2}$ has been introduced in eqs. (2.16) and 2.17). Note that, as in sect. 4.3 the coboundary of $\omega^{1}$ is a cup product in real Deligne cohomology:

$$
r_{2}\left(\left(\gamma_{1} \gamma_{2}\right)^{\prime}, \gamma_{2}^{\prime}\right)=\left(d \log \left(\gamma_{1} \gamma_{2}\right)^{\prime}, \log \left|\left(\gamma_{1} \gamma_{2}\right)^{\prime}\right|\right) \cup\left(d \log \gamma_{2}^{\prime}, \log \left|\gamma_{2}^{\prime}\right|\right) .
$$

where the two classes are associated to the rational functions $\left(\gamma_{1} \gamma_{2}\right)^{\prime}$ and $\gamma_{2}^{\prime}$, respectively (cf. sect. 2.4). Hence, we can work with the double complex of group cochains on $\Gamma$ with values in the real Deligne complex $\widetilde{\mathbb{R}(2)})_{\mathcal{D}}^{\bullet} \stackrel{\simeq}{\rightarrow} \underline{\varepsilon}_{U_{\Gamma}}^{\bullet}(1)$ on the region of discontinuity $U_{\Gamma}$. Since from this point on only rational functions with singularities at certain prescribed points will appear, following ref. [21] we will consider the Deligne complex on the generic point $\eta_{\mathbb{P}^{1}}$ of $\mathbb{P}^{1}$.

For any two elements $\gamma_{i}, \gamma_{j} \in \Gamma$ define $T_{i j} \in \mathrm{PSL}_{2}(\mathbb{C})$ by

$$
z \longmapsto T_{i j}(z)=\left[z: z_{i j}: z_{j}: \infty\right]=\frac{z-z_{i j}}{z-z_{j}} .
$$

where $z_{j}=\gamma_{j}^{-1}(\infty)$ and $z_{i j}=\gamma_{j}^{-1}\left(z_{i}\right)$. Following ref. [30], we introduce the 1-cochain on $\Gamma$ with values in $\underline{\mathcal{E}}_{\mathbb{P}^{1}}^{1}(1)\left(\eta_{\mathbb{P}^{1}}\right):$

$$
\kappa_{\gamma}=\log \left|c_{\gamma}\right| \pi_{1} d \log \gamma^{\prime} .
$$

We have: 
Lemma 4.7. $r_{2}\left(\left(\gamma_{1} \gamma_{2}\right)^{\prime}, \gamma_{2}^{\prime}\right)=4 d\left(\mathscr{L}_{2} \circ T_{12}\right)+\check{\delta} \kappa_{\gamma_{1}, \gamma_{2}}$, where $\mathscr{L}_{2}=\sqrt{-1} \mathscr{D}_{2}$, and $\mathscr{D}_{2}$ is the standard Bloch-Wigner dilogarithm, cf. 2.4.

Proof. A straightforward calculation exploiting relation (B.3).

Since obviously $d \kappa_{\gamma}=0$, we can redefine $\omega_{\gamma}^{1} \rightarrow \omega_{\gamma}^{1}+\kappa_{\gamma}$, so that using the lemma, from eq. (4.21) we have:

$$
\check{\delta}\left(\omega^{1}[\log \rho]\right)_{\gamma_{1}, \gamma_{2}}=-4 d\left(\mathscr{L}_{2} \circ T_{12}\right) .
$$

For convenience of notation, let us temporarily set $\hat{\omega}_{\gamma_{1}, \gamma_{2}}^{2}:=-4\left(\mathscr{L}_{2} \circ T_{12}\right)$. We then have the following

\section{Lemma 4.8.}

$$
D\left(\omega^{0}+\omega^{1}+\hat{\omega}^{2}\right)=-4 \mathscr{L}_{2}\left(\left[\infty: \gamma_{1}(\infty): \gamma_{1} \gamma_{2}(\infty): \gamma_{1} \gamma_{2} \gamma_{3}(\infty)\right]\right) .
$$

Proof. By construction, $D\left(\omega^{0}+\omega^{1}+\hat{\omega}^{2}\right)=\check{\delta} \hat{\omega}^{2}$, and the latter Čech coboundary is computed as

$$
\begin{aligned}
\check{\delta}_{\hat{\omega}_{1}, \gamma_{2}, \gamma_{3}}^{2}=- & 4\left(\mathscr{L}_{2}\left(\left[z: z_{23}: z_{3}: \infty\right]\right)-\mathscr{L}_{2}\left(\left[z: z_{123}: z_{3}: \infty\right]\right)\right. \\
& \left.+\mathscr{L}_{2}\left(\left[z: z_{123}: z_{23}: \infty\right]\right)-\mathscr{L}_{2}\left(\left[\gamma_{3}(z): z_{12}: z_{2}: \infty\right]\right)\right)
\end{aligned}
$$

In the last term in the previous relation we have $\left[\gamma_{3}(z): z_{12}: z_{2}: \infty\right]=\left[z: z_{123}: z_{23}: z_{3}\right]$. The Bloch-Wigner dilogarithm satisfies the 5 -term relation [15, 23]:

$$
\sum_{i=0}^{4}(-1)^{i} \mathscr{D}_{2}\left(\left[a_{0}: \cdots: \hat{a_{i}}: \cdots: a_{4}\right]\right)=0,
$$

where $a_{0}, \ldots, a_{4} \in \mathbb{P}^{1}$ and the hat sign denotes omission. As a consequence we have:

$$
\check{\delta} \hat{\omega}_{\gamma_{1}, \gamma_{2}, \gamma_{3}}^{2}=-4 \mathscr{L}_{2}\left(\left[z_{123}: z_{23}: z_{3}: \infty\right]\right),
$$

and again by the invariance of the cross ratio, we obtain 4.25).

Remark 4.9. $\mathscr{D}_{2}([a: b: c: d])$ is the hyperbolic volume of the ideal hyperbolic tetrahedron with vertices at the points $a, b, c, d \in \mathbb{P}^{1}$, see, e.g. refs. [16, 14, 23].

It follows from the five-term relation (4.26) that the right hand side of eq. (4.25) defines an $\mathbb{R}(1)$-valued 3cocycle on $\Gamma$. Moreover, this cocycle is already defined on $\mathrm{PSL}_{2}(\mathbb{C})$, where its class is known to generate the Eilenber-Mac Lane cohomology group $H^{3}\left(\mathrm{PSL}_{2}(\mathbb{C}), \mathbb{R}(1)\right)$, 7] 14, 23. It is also known that up to a factor 2 it agrees with the imaginary part of the second Cheeger-Simons universal secondary class $\hat{C}_{2}$.

Thus the complete Liouville cocycle $\Omega=\omega^{0}+\omega^{1}+\omega^{2}$ subordinated to the cover $U_{\Gamma} \rightarrow X$ is found as follows. The pullback of $\hat{C}_{2}$ along the inclusion map $\Gamma \hookrightarrow \mathrm{PSL}_{2}(\mathbb{C})$ is zero, since ( $\Gamma$ being of second kind) the 3-manifold $M_{\Gamma}=\mathbb{H}^{3} / \Gamma$ is non-compact, and $H^{\bullet}(\Gamma, \mathbb{R}(1)) \cong H^{\bullet}\left(M_{\Gamma}, \mathbb{R}(1)\right)$. It follows that the restriction of the cocycle given by the Bloch-Wigner dilogarithm to $\Gamma$ must be a coboundary, hence there exists a group 2-cochain $c$ on $\Gamma$ with values in $\mathbb{R}(1)$ such that

$$
4 \mathscr{L}_{2}\left(\left[\infty: \gamma_{1}(\infty): \gamma_{1} \gamma_{2}(\infty): \gamma_{1} \gamma_{2} \gamma_{3}(\infty)\right]\right)=(\check{\delta} c)_{\gamma_{1}, \gamma_{2}, \gamma_{3}}
$$

It follows that the cochain $c$ provides the necessary "integration constants," namely the required 2-cochain on $\Gamma$ with values in $\underline{\varepsilon}_{U_{\Gamma}}^{0}$ to complete the Liouville cocycle is $\omega_{\gamma_{1}, \gamma_{2}}^{2}=-4\left(\mathscr{L}_{2} \circ T_{12}\right)+c_{\gamma_{1}, \gamma_{2}}$.

\section{Conformal metrics and hermitian holomorphic cohomology}

In sect. 4 we presented a construction of a degree $2, \mathbb{R}(1)$-valued class corresponding to a conformal metric $\rho \in$ $\mathscr{C} \mathscr{M}(X)$, represented by the cocycle $\Omega[\log \rho]$. Supplemented by the area of $X$ computed with respect to $\rho$, it provides a global functional for the variational problem associated to the Liouville equation (4.2). We now show that it coincides with the square of the class of $\left(T_{X}, \rho\right)$ in hermitian holomorphic Deligne cohomology introduced in sect. 3 Moreover, we show that this equality holds at the cocycle level. More generally, without considering the area term, we show that the cup product of $(L, \rho)$ and $\left(L^{\prime}, \rho^{\prime}\right)$ in hermitian holomorphic Deligne cohomology coincides with the class of $\Omega\left[\log \rho, \log \rho^{\prime}\right]$, and again the equality in fact holds at the cocycle level. 


\subsection{Comparison on a curve}

Let $X$ be a compact Riemann surface. From the results of sect. 3.3 the cup product of the classes of $(L, \rho)$ and $\left(L^{\prime}, \rho^{\prime}\right)$ in hermitian holomorphic Deligne cohomology yields a class in $H_{\mathcal{D}_{h . h .}}^{4}(X, 2)$, and on a curve we only capture the 2-dimensional part of this class. Indeed, in the exact sequence (3.6), the cohomology class corresponding to the symbol $\left(L, L^{\prime}\right] \in H_{\mathcal{D}}^{4}(X, \mathbb{Z}(2))$ is zero, and $A^{(2,2)}(X)_{\mathbb{R}(2)}$ is also zero for obvious dimensional reasons, so we have:

$$
\cdots \longrightarrow H_{\mathcal{D}}^{3}(X, \mathbb{R}(2)) \longrightarrow H_{\mathcal{D}_{h . h .}}^{4}(X, 2) \longrightarrow 0 .
$$

It follows that the class $[L, \rho] \cup\left[L^{\prime}, \rho^{\prime}\right]$ must come from an element in $H_{\mathcal{D}}^{3}(X, \mathbb{R}(2))$. As already remarked, on a curve we have $\widetilde{\mathbb{R}(2)})_{\mathcal{D}}^{\bullet} \stackrel{\simeq}{\rightarrow} \underline{\varepsilon}_{X}^{\bullet}(1)[-1]$, thus $H_{\mathcal{D}}^{3}(X, \mathbb{R}(2)) \cong \mathbf{H}^{3}\left(X, \underline{\varepsilon}_{X}^{\bullet}(1)[-1]\right) \cong \mathbf{H}^{2}\left(X, \underline{\varepsilon}_{X}^{\bullet}(1)\right) \cong H^{2}(X, \mathbb{R}(1))$, in agreement with the calculations performed in sect. [4

More in detail, in complex dimension 1 the second hermitian holomorphic Deligne complex $D(1)_{h . h .}^{\bullet}$ simplifies considerably and diagram (3.17) reduces to

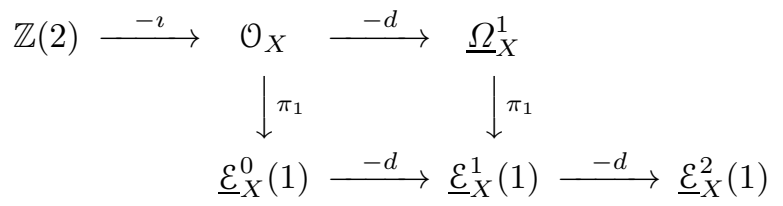

so that $D(2)_{h}^{\bullet}$.h. becomes just the cone of the morphism $\mathbb{Z}(2)_{\mathcal{D}}^{\bullet} \stackrel{\pi_{1}}{\longrightarrow} \underline{\mathcal{E}}_{X}^{\bullet}(1)[-1]$. In other words, on a curve $X$ we have that $D(2)_{h . h}^{\bullet}$ is given by the complex

$$
\mathbb{Z}(2)_{X} \stackrel{-\imath}{\longrightarrow} \mathcal{O}_{X} \stackrel{\left(-d,-\pi_{1}\right)}{\longrightarrow} \underline{\Omega}_{X}^{1} \oplus \underline{\varepsilon}_{X}^{0}(1) \stackrel{-\pi_{1}+d}{\longrightarrow} \underline{\varepsilon}_{X}^{1}(1) \stackrel{d}{\longrightarrow} \underline{\varepsilon}_{X}^{2}(1),
$$

where the differentials have been written explicitly. We can see the complex $\underline{\varepsilon}_{X}^{\bullet}$ appears as a subcomplex in (5.2) and the shift of two positions to the right clearly accounts for the cohomology degree shift from 2 to 4 .

Our main result is the following comparison

Theorem 5.1. Let $X$ be a compact Riemann surface of genus $g>1$. Let $(L, \rho)$ and $\left(L^{\prime}, \rho^{\prime}\right)$ be two hermitian holomorphic line bundles. The class of $[L, \rho] \cup\left[L^{\prime}, \rho^{\prime}\right]$ in $H_{\mathcal{D}_{h . h .}^{4}}(X, 2) \cong H^{2}(X, \mathbb{R}(1))$ coincides with the one represented by the cocycle $\Omega\left[\log \rho, \log \rho^{\prime}\right]$ constructed in section 母.

Proof. We have observed above that $H_{\mathcal{D}_{h . h}}^{4}(X, 2) \cong H^{2}(X, \mathbb{R}(1))$, and by construction the class of $\Omega\left[\log \rho, \log \rho^{\prime}\right]$ is in $\mathbf{H}^{2}\left(X, \underline{\varepsilon}_{X}^{\bullet}(1)\right) \cong H^{2}(X, \mathbb{R}(1))$. Note that for $X$ connected they must coincide up to a proportionality factor, since $H^{2}(X, \mathbb{R}(1)) \cong \mathbb{R}(1)$ in this case. In general, we compute the proportionality factor from the explicit cocycles from sects. 3.3 and 4.2 to 4.4 (Since $\Omega\left[\log \rho, \log \rho^{\prime}\right]$ is computed under suitable acyclicity assumptions on the cover, so we will use such a cover to establish the comparison.)

Let us assume $L$ and $L^{\prime}$ and their respective hermitian metric structures are represented by cocycles of type (3.14) with respect to the chosen cover $\mathfrak{U}_{X}$. Specializing the general expression in table 3.18 in sect. 3.3 to the case at hand we obtain, with reference to (5.2):

\begin{tabular}{cc}
$(0,4)$ & $c_{i j k} c_{k l m}^{\prime}$ \\
\hline$(1,3)$ & $-c_{i j k} \log g_{k l}^{\prime}$ \\
\hline$(2,2)$ & $-\log g_{i j} d \log g_{j k}^{\prime} \oplus-\pi_{1} \log g_{i j} \log \left|g_{j k}^{\prime}\right|$ \\
\hline$(3,1)$ & $(1-\alpha)\left(d^{c} \log \left|g_{i j}\right| \frac{1}{2} \log \rho_{j}^{\prime}-\log \left|g_{i j}\right| \frac{1}{2} d^{c} \log \rho_{j}^{\prime}\right)+\alpha\left(-\frac{1}{2} d^{c} \log \rho_{i} \log \left|g_{i j}^{\prime}\right|+\frac{1}{2} \log \rho_{i} d^{c} \log \left|g_{i j}^{\prime}\right|\right)$ \\
\hline$(4,0)$ & $\alpha \bar{\partial} \partial \log \rho_{i} \frac{1}{2} \log \rho_{i}^{\prime}+(1-\alpha) \frac{1}{2} \log \rho_{i} \bar{\partial} \partial \log \rho_{i}^{\prime}$
\end{tabular}

where we have followed the convention explained in the introduction for the bidegrees. Let us denote by $\theta^{i}$ the term of bidegree $(4-i, i)$ in (5.3) and by $\Theta$ the total cocycle. (For simplicity, we suppress $\rho$ and $\rho^{\prime}$ from the notation.) A direct calculation shows that

$$
\theta_{i}^{0}=\omega_{i}^{0}+d \lambda_{i}^{0}, \quad \theta_{i j}^{1}=\omega_{i j}^{1}-\check{\delta} \lambda_{i j}^{0},
$$

where $\omega_{i}^{0}$ is given by eq. (4.15), $\omega^{1}$ is given by eq. (4.16), and

$$
\lambda_{i}^{0}=\alpha \frac{1}{2} d^{c} \log \rho_{i} \log \rho_{i}^{\prime}+(1-\alpha) \frac{1}{2} \log \rho_{i} d^{c} \log \rho_{i}^{\prime} .
$$


Note that at this point we could simply define $\Omega=\Theta-D \lambda^{0}$. Furthermore, note that $\Omega$ does not explicitly depend on the parameter $\alpha$ from the Beilinson product (2.5). To finish the comparison, let us assume the cover $\mathcal{U}_{X}$ allows us to find a collection $L_{i j k} \in \mathcal{O}_{X}\left(U_{i j k}\right)$ such that

$$
\begin{aligned}
d L_{i j k} & =-\log g_{i j} d \log g_{j k}^{\prime} \\
\check{\delta} L_{i j k l} & =-c_{i j k} \log g_{k l}^{\prime}+n_{i j k l},
\end{aligned}
$$

as in sect. 4.3 In this way we have $\Omega=\left(\omega^{0}, \omega^{1}, \omega^{2}\right)$ with $\omega_{i j k}^{2}=-\mathscr{L}_{2}\left(g_{i j}, g_{j k}^{\prime}\right) . \Omega$ is a cocycle of total degree 2 in $\operatorname{Tot} \check{C}^{\bullet}\left(\mathcal{U}_{X}, \underline{\varepsilon}_{X}^{\bullet}\right)$, and it injects (via the exact sequence of the cone) into Tot $\check{C}^{\bullet}\left(\mathcal{U}_{X}, D(2){ }_{h . h .}\right)$ as

$$
\left(\omega^{0}, \omega^{1}, 0 \oplus \omega^{2}\right) .
$$

Then via equations (5.4) it is easily seen that

$$
\theta_{i j k}^{2}=d_{D(2))_{h . h .}}\left(-L_{i j k}\right)+0 \oplus \omega_{i j k}^{2},
$$

where $d_{D(2)} \boldsymbol{\bullet}_{h . h .}$ is the differential in $D(2)_{h . h .}^{\bullet}$, and therefore

$$
\Theta=\left(\omega^{0}, \omega^{1}, 0 \oplus \omega^{2}\right)+D \lambda^{0}+D(-L, n),
$$

where we have put $D=d_{D(2))_{h . h .}} \pm \check{\delta}$ for the total differential. Thus the two cocycles constructed via the direct method of sect. 4.2 and the cup product of metrized bundles define the same class. By direct comparison, the proportionality factor is 1 .

In light of the previous theorem, the polarization identity in Remark 4.3 is now easily explained. Using $[L \otimes$ $\left.L^{\prime}, \rho \rho^{\prime}\right]=[L, \rho]+\left[L^{\prime}, \rho^{\prime}\right]$ and $\left[L \otimes L^{\prime \vee}, \rho / \rho^{\prime}\right]=[L, \rho]-\left[L^{\prime}, \rho^{\prime}\right]$, and the (graded) commutativity of the cup product

$$
H_{\mathcal{D}_{h . h .}}^{2}(X, 1) \otimes H_{\mathcal{D}_{h . h .}}^{2}(X, 1) \stackrel{\cup}{\longrightarrow} H_{\mathcal{D}_{h . h .}}^{4}(X, 2),
$$

we obtain the polarization identity

$$
4[L, \rho] \cup\left[L^{\prime}, \rho^{\prime}\right]=\left[L \otimes L^{\prime}, \rho \rho^{\prime}\right]^{2}-\left[L \otimes L^{\prime \vee}, \rho / \rho^{\prime}\right]^{2},
$$

where the squares in the right hand side refer to $\cup$. A polarization identity at the level of representative cocycles, and hence the one in Remark 4.3 follow by applying Thm. 5.1] to the latter identity.

By choosing $L=L^{\prime}=T_{X}$, the holomorphic tangent bundle of $X$, we immediately obtain:

Corollary 5.2. Let $\rho \in \mathscr{C} \mathscr{M}(X)$ be a conformal metric. The Liouville functional without area term (4.6) is given by the (evaluation of) the square $\left[T_{X}, \rho\right] \cup\left[T_{X}, \rho\right]$ with respect to the cup product in hermitian holomorphic Deligne cohomology. The full-fledged Liouville functional is obtained by adding the area term $\frac{1}{2 \pi} \int_{X} \omega_{\rho}$ to (4.6).

Remark 5.3. Due to the specific form of the differential in the complex $D(2)_{h . h}^{\bullet}$. the descent equations are explicit and close automatically. Therefore the cocycle resulting from the calculation of the cup product sidesteps the problem of the explicit calculation of the last term, unlike the more direct version from sect. 4 Thus, thanks to the explicit character of the calculation, specific assumptions on the nature of the cover $\mathcal{U}_{X}$ are not required.

It follows from Thm 5.1 corollary 5.2 and the previous remark that definition 4.1 applies to any (étale) cover $\mathcal{U}_{X} \rightarrow X$. Indeed, proposition 4.4 from sect. 4.2 can be reformulated at the cocycle level as follows:

Proposition 5.4. Let $X$ be a compact, genus $g>1$ Riemann surface and let $\mathcal{U}_{X} \rightarrow X$ be a cover. For a conformal metric $\rho \in \mathscr{C} \mathscr{M}(X)$ and $\sigma \in C^{\infty}(X, \mathbb{R})$, there is a cocycle $\hat{\Omega}_{u_{X}}[\log \rho]$ solving the variational problem for the Liouville equation.

Proof. If $\rho$ is a conformal metric, let the pair $\left(T_{X}, \rho\right)$ be represented, as an hermitian line bundle, by a cocycle $c\left(T_{X}, \rho\right)$ with respect to the cover $\mathcal{U}_{X}$. We set

$$
\Omega[\log \rho]=c\left(T_{X}, \rho\right) \cup c\left(T_{X}, \rho\right),
$$

and a simple calculation starting from eq. (5.3) yields

$$
\Omega[\log \rho+\sigma]-\Omega[\log \rho]=\sigma c_{1}(\rho)+\frac{1}{2} \sigma \partial \bar{\partial} \sigma+D \chi,
$$


where $\chi_{i}=\frac{1}{2} \sigma \frac{1}{2} d^{c} \log \rho_{i}-\frac{1}{2} d^{c} \sigma \frac{1}{2} \log \rho_{i}$. Now define

$$
\hat{\Omega}_{\mathcal{U}_{X}}[\log \rho]=\Omega[\log \rho]-\sqrt{-1} \omega_{\rho} .
$$

We see that it yields the formula in Proposition 4.4 In particular we have that

$$
\left.\frac{d}{d t}\right|_{t=0} \hat{\Omega}_{u_{X}}[\log \rho+t \sigma] \equiv \sigma\left(c_{1}(\rho)-\sqrt{-1} \omega_{\rho}\right),
$$

where $\equiv$ means "up to total coboundary."

\subsection{Determinant of cohomology}

Let again $L$ and $L^{\prime}$ be two holomorphic line bundles with hermitian metrics $\rho$ and $\rho^{\prime}$, respectively, on the compact Riemann surface $X$. Brylinski proves in 10] that the cup product of $L$ and $L^{\prime}$ in hermitian holomorphic Deligne cohomology yields the (logarithm) of the metric $\|\cdot\|$ on the Deligne pairing $\left\langle L, L^{\prime}\right\rangle$ defined in [1].

It follows, via Thm 5.1 and the isomorphism 3.2 between our version of hermitian holomorphic cohomology and Brylinski's, that the class of $\Omega\left[\log \rho, \log \rho^{\prime}\right]$ is also equal to $\log \left\|\left\langle L, L^{\prime}\right\rangle\right\|$. It is worthwhile to provide a direct proof of this fact starting from the explicit cocycle given in $[5.3$.

First, we need to recall a few definitions from 11. A complex line $\left\langle L, L^{\prime}\right\rangle$ is assigned to the pair $\left(L, L^{\prime}\right)$ as follows. Let $D$ and $D^{\prime}$ be divisors on $X$ corresponding to $L$ and $L^{\prime}$, and assume they have disjoint supports. Consider two rational sections, $s$ and $s^{\prime}$ such that $(s)=D$ and $\left(s^{\prime}\right)=D^{\prime}$. To this datum one assigns a copy of the complex line generated by the symbol $\left\langle s, s^{\prime}\right\rangle$ subject to the relations:

$$
\begin{aligned}
& \left\langle f s, s^{\prime}\right\rangle=f\left(D^{\prime}\right)\left\langle s, s^{\prime}\right\rangle \\
& \left\langle s, g s^{\prime}\right\rangle=g(D)\left\langle s, s^{\prime}\right\rangle
\end{aligned}
$$

whenever $f$ is a rational function with divisor $(f)$ disjoint from $D^{\prime}$, and similarly for $g$. The Weil reciprocity relation $f(\operatorname{div}(g))=g(\operatorname{div}(f))$ (cf. ref. 22) for two rational functions $f$ and $g$ with disjoint divisors implies that the relations (5.5) are consistent and the complex line depends only on the pair $L, L^{\prime}$. When the line bundles are equipped with hermitian metrics, generically denoted by $\|\cdot\|$, the assignment ${ }^{5}$

$$
\log \left\|\left\langle s, s^{\prime}\right\rangle\right\|^{2}=\frac{1}{2 \pi \sqrt{-1}} \int_{X} \partial \bar{\partial} \log \|s\|^{2} \log \left\|s^{\prime}\right\|^{2}+\log \|s\|^{2}\left[D^{\prime}\right]+\log \left\|s^{\prime}\right\|^{2}[D]
$$

is compatible with the relations (5.5) and defines an hermitian metric on the complex line $\left\langle L, L^{\prime}\right\rangle$. In formula (5.6) the operator $\partial \bar{\partial}$ is to be computed in the sense of distributions.

Having covered the main definitions, we can now state

Theorem 5.5. The cup product of $(L, \rho)$ with $\left(L^{\prime}, \rho^{\prime}\right)$ in hermitian holomorphic Deligne cohomology corresponds to the logarithm of the norm (5.6) on the Deligne pairing $\left\langle L, L^{\prime}\right\rangle$. The proportionality factor is $-\pi \sqrt{-1}$.

Proof. Let $D$ and $D^{\prime}$ be divisors with disjoint support on $X$ corresponding to $L$ and $L^{\prime}$, respectively. Using the same technique as in refs. [10, 11, consider two $C^{\infty}$ positive real functions $f_{1}$ and $f_{2}$ such that $f_{1}+f_{2}=1$ and $f_{1}($ resp. $\left.f_{2}\right)$ vanishes in a neighborhood of the support of $D^{\prime}$ (resp. $\left.D\right)$. Also, set $U_{1}=X \backslash \operatorname{supp}\left(D^{\prime}\right)$ and $U_{2}=X \backslash \operatorname{supp}(D)$. Thus $\left\{f_{1}, f_{2}\right\}$ is just a partition of unity subordinated to the cover $\mathcal{U}_{X}=\left\{U_{1}, U_{2}\right\}$.

The only two terms different from zero in the cocycle $\Theta$ in (5.3) representing the class $[L, \rho] \cup\left[L^{\prime}, \rho^{\prime}\right]$ with respect to this cover are $\theta_{i}^{0}$ and $\theta_{i}^{1}$, with the Cech index $i \in\{1,2\}$. Thus the class we are after is equivalently given by the integral

$$
\int_{X} f_{1} \theta_{1}^{0}+f_{2} \theta_{2}^{1}+d f_{2} \wedge \theta_{21}^{1}
$$

which is arrived at by applying in the standard homotopy operator based on the partition of unity $\left\{f_{1}, f_{2}\right\}$ : from $\check{\delta} \theta_{12}^{1}=0$ we obtain that $\theta_{12}^{1}$ is the coboundary of the cochain $j \rightarrow \sum_{i=1,2} f_{i} \theta_{i j}^{1}$ and then we use $\theta_{2}^{0}-\theta_{2}^{0}=-d \theta_{12}^{1}$. Observe that the 2-form in (5.7) is globally well defined over $X$ : $\theta_{21}^{1}$ is defined only on $U_{1} \cap U_{2}$, but $d f_{2}$ has support on $U_{1} \cap U_{2}$, so their wedge product is defined everywhere; similarly, $f_{i} \theta_{i}^{0}$ is everywhere defined thanks to the fact that $f_{i}$ has support in $U_{i}, i=1,2$.

\footnotetext{
${ }^{5}$ We write the square explicitly, whereas the symbol $\|\cdot\|$ used in ref. [11 denotes the square of the norm.
} 
Consider rational sections $s$ and $s^{\prime}$ of $L$ and $L^{\prime}$ such that $\operatorname{div}(s)=D$ and $\operatorname{div}\left(s^{\prime}\right)=D^{\prime}$ as above. With respect to the two-element cover $\mathfrak{U}_{X}=\left\{U_{1}, U_{2}\right\}$, the section $s$ corresponds to the pair $\left\{s_{1}, s_{2}\right\}$, and similarly for $s^{\prime}=\left\{s_{1}^{\prime}, s_{2}^{\prime}\right\}$. Since $\operatorname{supp}(D)$ is contained in $U_{1}$ but not in $U_{2}$, and the other way around for $D^{\prime}$, it follows that $s_{2}$ and $s_{1}^{\prime}$ are actually invertible functions over their respective domains. Following [25], we can assume that $s$ and $s^{\prime}$ are in fact the rational section $\mathbf{1}$, so that $s_{2}=1$ and $s_{1}^{\prime}=1$, and therefore:

$$
|s|_{\rho}^{2}= \begin{cases}\log \rho_{1}+\log \left|s_{1}\right|^{2} & \text { on } U_{1}, \\ \log \rho_{2} & \text { on } U_{2},\end{cases}
$$

and

$$
\left|s^{\prime}\right|_{\rho^{\prime}}^{2}= \begin{cases}\log \rho_{1}^{\prime} & \text { on } U_{1}, \\ \log \rho_{2}^{\prime}+\log \left|s_{2}^{\prime}\right|^{2} & \text { on } U_{2}\end{cases}
$$

Furthermore, $\log \left|g_{21}^{\prime}\right|=\log \left|s_{2}^{\prime}\right|$ on $U_{1} \cap U_{2}$. Let us denote by $\|\cdot\|=|\cdot|_{\rho}=|\cdot|_{\rho^{\prime}}$ for simplicity. Using the relevant entries from (5.3) we have:

$$
\begin{gathered}
\theta_{1}^{0}=c_{1}(\rho) \log \left\|s^{\prime}\right\|, \\
\theta_{2}^{0}=\bar{\partial} \partial \log \rho_{2} \frac{1}{2} \log \rho_{2}^{\prime}=d d^{c} \log \|s\| \frac{1}{2} \log \rho_{2}^{\prime} .
\end{gathered}
$$

An elementary integration by parts leads to:

$$
\begin{aligned}
\int_{X} f_{2} \theta_{2}^{0} & =\int_{X} f_{2} \log \|s\| c_{1}\left(\rho^{\prime}\right) \\
& +\int_{X} \log \|s\| d f_{2} \wedge \frac{1}{2} d^{c} \log \rho_{2}^{\prime}-\int_{X} \frac{1}{2} \log \rho_{2}^{\prime} d f_{2} \wedge d^{c} \log \|s\| .
\end{aligned}
$$

On the other hand, we have

$$
\int_{X} d f_{2} \wedge \theta_{12}^{1}=\int_{X} \log \|s\| d f_{2} \wedge d^{c} \log \left|s_{2}^{\prime}\right|-\int_{X} \log \left|s_{2}^{\prime}\right| d f_{2} \wedge d^{c} \log \|s\|,
$$

and putting all terms together we obtain

$$
\begin{aligned}
\int_{X} f_{1} \theta_{1}^{0}+f_{2} \theta_{2}^{1}+d f_{2} \wedge \theta_{21}^{1} & =\int_{X} f_{1} c_{1}(\rho) \log \left\|s^{\prime}\right\|+\int_{X} f_{2} \log \|s\| c_{1}\left(\rho^{\prime}\right) \\
& +\int_{X} \log \|s\| d f_{2} \wedge d^{c} \log \left\|s^{\prime}\right\|+\int_{X} \log \left\|s^{\prime}\right\| d f_{1} \wedge d^{c} \log \|s\|
\end{aligned}
$$

which, if expressed in terms of the squares of the norms, is (up to a factor) the logarithm of $\left\|\left\langle s, s^{\prime}\right\rangle\right\|$, as it is found in [11, formula 6.5.1]. This version is due to O. Gabber. Via the Poincaré-Lelong lemma (see, e.g. [22])

$$
\bar{\partial} \partial \log \|s\|=c_{1}(\rho)+2 \pi \sqrt{-1}[D],
$$

where $[D]$ is the delta-current supported at the divisor of $s$, and similarly for $s^{\prime}$, formula (5.8) can be recast into:

$$
\begin{aligned}
\int_{X} f_{1} \theta_{1}^{0}+f_{2} \theta_{2}^{1}+d f_{2} \wedge \theta_{21}^{1} & =\int_{X} d d^{c} \log \|s\| \log \left\|s^{\prime}\right\|-2 \pi \sqrt{-1} \log \|s\|\left[D^{\prime}\right]-2 \pi \sqrt{-1} \log \left\|s^{\prime}\right\|[D] \\
& =-2 \pi \sqrt{-1} \log \left\|\left\langle s, s^{\prime}\right\rangle\right\|
\end{aligned}
$$

which is what we wanted to show.

This allows us to recast the Liouville functional for conformal metrics on $X$ in the following form.

Corollary 5.6. The exponential of the Liouville functional defines an hermitian metric on the complex line $\left\langle T_{X}, T_{X}\right\rangle$, namely for a conformal metric $\rho \in \mathscr{C} \mathscr{M}(X)$ we have:

$$
\exp S[\log \rho]=\left\|\left\langle T_{X}, T_{X}\right\rangle\right\| \exp \frac{1}{2 \pi} A_{X}(\rho),
$$

where $A_{X}(\rho)$ is the area of $X$ with respect to $\rho$. 
Remark 5.7. The above corollary justifies the choice made in Definition 4.1 for the various factors $2 \pi \sqrt{-1}$.

A similar result has been obtained in ref. 32] by considering the Liouville action functional defined on the Schottky space, and in fact the results in loc. cit. are formulated in terms of a Schottky family.

Indeed, the statement in Corollary [5.6 can be immediately reformulated for a family $\pi: X \rightarrow S$ with base parameter space $S$ by considering the relative holomorphic tangent line bundle $T_{X / S}$ with an hermitian fiber metric $\rho$. (Thus $\rho_{s} \in \mathscr{C} \mathscr{M}\left(X_{s}\right)$ for every fiber $X_{s}, s \in S$.) Notice that the fiber metric $\rho$ needs not be critical (i.e. satisfying the fiberwise constant negative curvature condition).

\section{Conclusions and outlook}

In this paper we have provided a geometric description of the construction of the Liouville action functional for constant negative curvature metrics on compact Riemann surfaces of genus $g \geq 2$.

Our approach was to construct a Hermitian holomorphic Deligne cohomology class as the cup square of the metrized holomorphic tangent bundle $T_{X}$, and then show at the level of cocycles that (modulo an area term) this construction agrees with those in [30] and earlier works.

Furthermore, our construction leads to the identification of the class corresponding to the Liouville action with the determinant of cohomology construction. Hence it could serve as an alternative construction of the latter in terms of different choices of cocycles.

One of the most important properties of the Liouville functional from the works [33, 34, 32 is the link with the Weil-Petersson geometry of the Teichmüller space. From this point of view, an analysis of the behavior of the Liouville action for families of Riemann surfaces is crucial. In particular, a delicate analytic computation of the variation of the Action with respect to the moduli was carried out in the afore mentioned works to establish the link with the Weil-Petersson metric.

We have not pursued these matters in the present work limiting ourselves to establish the existence of a class for a metrized bundle on the base of a family $\pi: X \rightarrow S$. A more precise analysis would require, not only a more explicit description of the map $\int_{\pi}$ (also advocated in [9]), but a full computation of the Leray sequence associated to the family $\pi$. This is instrumental in defining relative Hermitian holomorphic classes, and in analyzing their variation with respect to base parameters.

We hope to pursue this and other problems related to the extension of the present work to singular metrics in a different publication.

\section{A Cones}

In the main body of the paper we have used iterated cones to define the hermitian holomorphic Deligne complexes. One technical problem one has to face concerns the homotopy (graded) commutativity of the modified Beilinson product defined in eq. (2.5). A problem arises because the factors in the cones are cones themselves and therefore they have multiplication structures which are graded commutative up to homotopy to begin with. We want to show that even in this situation the final resulting product on cones is again homotopy graded commutative. This ensures that on cohomology the product will be genuinely graded commutative, so that in particular hermitian holomorphic Deligne cohomology as defined in section 3 has the correct product structure.

\section{A.1 Cones and homotopies}

We consider the following situation. For $i=1,2,3$ we have maps of complexes: $f_{i}: A_{i}^{\bullet} \rightarrow B_{i}^{\bullet}$, and for $i<j$ maps $a_{j i}: A_{i}^{\bullet} \rightarrow A_{j}^{\bullet}$ and $b_{j i}: B_{i}^{\bullet} \rightarrow B_{j}^{\bullet}$. Also, let $C^{\bullet}\left(f_{i}\right)=\operatorname{Cone}\left(f_{i}: A_{i}^{\bullet} \rightarrow B_{i}^{\bullet}\right)$, for $i=1,2,3$.

First, consider the homotopy commutative diagram:

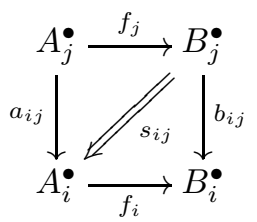

where $s_{i j}: A_{j}^{\bullet} \rightarrow B_{i}^{\bullet-1}$ is the homotopy map of complexes:

$$
f_{i} a_{i j}-b_{i j} f_{j}=d s_{i j}+s_{i j} d .
$$


An immediate verification yields

Lemma A.1. The diagram (A.1) can be extended to

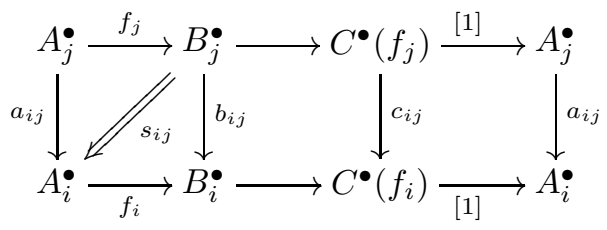

where the map $c_{i j}$ is given by $\left(\begin{array}{cc}a_{i j} & 0 \\ -s_{i j} & b_{i j}\end{array}\right)$ and the squares containing the cones are in fact strictly commutative.

Remark A.2. This lemma is nothing other than the statement that any homotopy commutative diagram of the form (A.1) in the category of complexes in an abelian category can be extended to a (homotopy) commutative diagram of distinguished triangles, that is, one of the axioms defining a triangulated category, see, e.g. 31.

For $k<j<i$ consider the homotopy commutative triangle

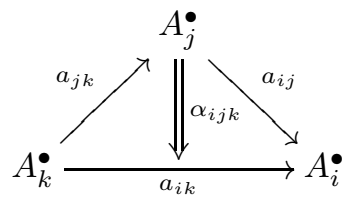

where $a_{i k}-a_{i j} a_{j k}=d \alpha_{i j k}+\alpha_{i j k} d$, and similarly for the complexes $B_{i}^{\bullet}$, with a corresponding homotopy $\beta_{i j k}$. Thus $\alpha_{i j k}: A_{k}^{\bullet} \rightarrow A_{i}^{\bullet-1}$ and $\beta_{i j k}: B_{k}^{\bullet} \rightarrow B_{i}^{\bullet-1}$. Now consider the diagram:

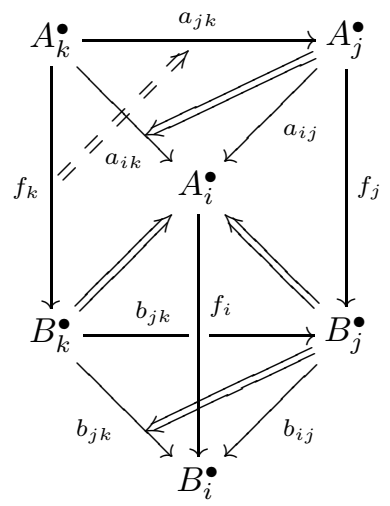

The faces in (A.2) are homotopy commutative, however we assume that composing the faces is strictly commutative, namely the two possible homotopies $b_{i j} b_{j k} f_{k} \Longrightarrow f_{i} a_{i k}$ must be equal. Concretely, this corresponds to the relation

$$
s_{i k}+\beta_{i j k} f_{k}=f_{i} \alpha_{i j k}+s_{i j} a_{j k}+b_{i j} s_{j k} .
$$

We have:

Lemma A.3. The map

$$
\left(\begin{array}{cc}
-\alpha_{i j k} & 0 \\
0 & \beta_{i j k}
\end{array}\right): C^{\bullet}\left(f_{k}\right) \longrightarrow C^{\bullet-1}\left(f_{i}\right)
$$

realizes the homotopy

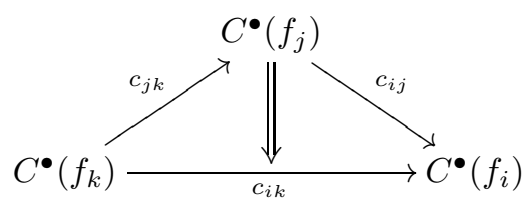

Proof. It is an elementary calculation based on writing $c_{i k}-c_{i k} c_{j k}$ explicitly via the matrix representation given in Lemma A.1 and using eq. A.3. 


\section{A.2 Applications}

Consider the same setup as in section 2.1 with the same complexes $X_{i}^{\bullet}$, etc., and diagrams:

$$
\mathcal{D}_{i} \stackrel{\text { def }}{=} X_{i}^{\bullet} \stackrel{f_{i}}{\longrightarrow} Z_{i}^{\bullet} \stackrel{g_{i}}{\longleftarrow} Y_{i}^{\bullet}
$$

from which we construct the cones

$$
C\left(\mathcal{D}_{i}\right)=\operatorname{Cone}\left(X_{i}^{\bullet} \oplus Y_{i}^{\bullet} \stackrel{f_{i}-g_{i}}{\longrightarrow} Z_{i}^{\bullet}\right)[-1], \quad i=1,2,3 .
$$

Moreover, following ref. [5], define $\mathcal{D}_{i} \otimes \mathcal{D}_{j}$ by taking the tensor product component-wise. Thus

$$
\mathcal{D}_{1} \otimes \mathcal{D}_{2}=X_{1}^{\bullet} \otimes X_{2}^{\bullet} \stackrel{f_{1} \otimes f_{2}}{\longrightarrow} Z_{1}^{\bullet} \otimes Z_{2}^{\bullet} \stackrel{g_{1} \otimes g_{2}}{\stackrel{\bullet}{\bullet}} Y_{1}^{\bullet} Y_{2}^{\bullet}
$$

Assuming as in section 2.1 that the product maps are compatible with the $f_{i}$, etc., the diagram

$$
\mathcal{D}_{1} \otimes \mathcal{D}_{2} \rightarrow \mathcal{D}_{3}
$$

is of the same type as A.1 and therefore lemma A.1 implies lemma 2.1

Now, let the multiplication maps $X_{1}^{\bullet} \otimes X_{2}^{\bullet} \rightarrow X_{3}^{\bullet}$ be graded commutative up to homotopy and similarly for the $Y_{i}^{\bullet}$ and the $Z_{i}^{\bullet}$. We are interested in the commutativity properties of multiplication map given by the Berlinson product (2.5).

Proposition A.4. The multiplication map $\cup_{\alpha}: C\left(\mathcal{D}_{1}\right) \otimes C\left(\mathcal{D}_{2}\right) \longrightarrow C\left(\mathcal{D}_{3}\right)$ given by (2.5) is homotopy graded commutative.

Proof. The permutation operation on tensor products induces the diagram

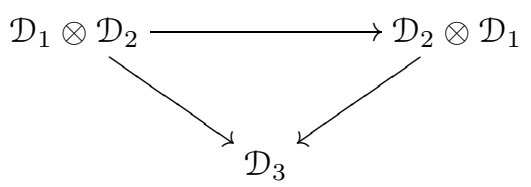

which is of type (A.2) and we can apply lemma A.3

It follows from the proposition that the cohomology inherits a well defined graded commutative product. This in particular applies to the definition of hermitian holomorphic Deligne cohomology that uses the cone (3.5). Therefore we conclude that the cup product (3.3) is graded commutative, as wanted.

\section{B Conventions on Kleinian groups and fractional linear transforma- tions}

As a reference the reader can consult, among many others, the book 26. Let $\Gamma$ be a finitely generated purely loxodromic non-elementary Kleinian group of the second kind, so there is a nonempty discontinuity region $U_{\Gamma} \subset \mathbb{P}^{1}$. The limit set is $L_{\Gamma}=\mathbb{P}^{1} \backslash U_{\Gamma}$. According to Ahlfors' finiteness theorem the quotient $X=U_{\Gamma} / \Gamma$ is a finite union of analytically finite Riemann surfaces. Thus:

$$
U_{\Gamma} / \Gamma=U_{1} / \Gamma_{1} \sqcup \cdots \sqcup U_{n} / \Gamma_{n}
$$

where $U_{1}, \ldots, U_{n}$ are the inequivalent components of $U_{\Gamma}$ and $\Gamma_{1}, \ldots, \Gamma_{n}$ their stabilizers. By way of example, a Schottky group has just one component, whereas a Fuchsian or quasi-Fuchsian group will have exactly two components.

We consider the map $U_{\Gamma} \rightarrow X$ as an étale cover, and Čech cohomology with respect to it translates into group cohomology for the group $\Gamma$, where the coefficient modules are sections over $U_{\Gamma}$ of the relevant sheaves. The group action is by pull-back. According to the conventions of ref. [3] we will write the coboundary operation as:

$$
(\check{\delta} c)_{\gamma_{1}, \ldots, \gamma_{n}}=c_{\gamma_{2}, \ldots, \gamma_{n}}+\sum_{i=1}^{n-1}(-1)^{i} c_{\gamma_{1}, \ldots, \gamma_{i} \gamma_{i+1}, \ldots, \gamma_{n}}+(-1)^{n}\left(c_{\gamma_{1}, \ldots, \gamma_{n-1}}\right) \cdot \gamma_{n}
$$


where $c$ is an $n$-cochain with values in some right $\Gamma$-module $A$. The expression $(\mathbb{B} .1)$ is the Cech coboundary applied to the nerve of the cover $U_{\Gamma} \rightarrow X$.

We assume that $\Gamma$ is normalized, namely the point $\infty$ belongs to the limit set $L_{\Gamma}$. If $\gamma \in \Gamma$ corresponds to the fractional linear transformation:

$$
\mathbb{P}^{1} \ni z \longmapsto \gamma(z)=\frac{a z+b}{c z+d},
$$

we have

$$
\gamma^{\prime}(z)=\frac{\operatorname{det} \gamma}{c^{2}\left(z-z_{\gamma}\right)^{2}}
$$

where $z_{\gamma}=-\frac{d}{c} \equiv \gamma^{-1}(\infty)$. Set

$$
c(\gamma) \equiv c_{\gamma} \stackrel{\operatorname{def}}{=} \frac{\operatorname{det} \gamma}{c^{2}} .
$$

The following properties are easily verified. If $z_{0}$ and $z_{\infty}$ are the attracting and repelling fixed points, respectively, then

$$
c_{\gamma}=\frac{\left(z_{0}-z_{\infty}\right)^{2} \lambda_{\gamma}}{\left(1-\lambda_{\gamma}\right)^{2}}
$$

where $\lambda_{\gamma}$ is the dilating factor. For $\gamma_{i}$ and $\gamma_{j}$ two elements of $\Gamma$, denote:

$$
z_{i}=\gamma^{-1}(\infty), \quad z_{i j}=\left(\gamma_{i} \gamma_{j}\right)^{-1}(\infty)=\gamma_{j}^{-1}\left(z_{i}\right), \quad c_{i}=c_{\gamma_{i}}, \quad c_{i j}=c_{\gamma_{i} \gamma_{j}} .
$$

We have the following relation:

$$
c_{\gamma_{1} \gamma_{2}}=\frac{c_{\gamma_{1}}}{c_{\gamma_{2}}}\left(z_{12}-z_{2}\right)^{2} .
$$

Finally, given four points $z_{1}, z_{2}, z_{3}, z_{4} \in \mathbb{P}^{1}$, we define their cross-ratio by:

$$
\left[z_{1}: z_{2}: z_{3}: z_{4}\right]=\frac{\left(z_{1}-z_{2}\right)\left(z_{3}-z_{4}\right)}{\left(z_{1}-z_{4}\right)\left(z_{3}-z_{2}\right)} .
$$

\section{References}

[1] Ettore Aldrovandi, Homological algebra of multivalued action functionals, Lett. Math. Phys. 60 (2002), no. 1, 47-58, arXiv:math-ph/0112031.

[2] Ettore Aldrovandi and Leon A. Takhtajan, Generating functional in CFT and effective action for twodimensional quantum gravity on higher genus Riemann surfaces, Comm. Math. Phys. 188 (1997), no. 1, $29-67$.

[3] _ Generating functional in CFT on Riemann surfaces. II. Homological aspects, Comm. Math. Phys. 227 (2002), no. 2, 303-348, arXiv:math.AT/0006147.

[4] A. A. Bêllinson, Higher regulators and values of L-functions, Current problems in mathematics, Vol. 24, Itogi Nauki i Tekhniki, Akad. Nauk SSSR Vsesoyuz. Inst. Nauchn. i Tekhn. Inform., Moscow, 1984, pp. 181-238.

[5] _ Notes on absolute Hodge cohomology, Applications of algebraic $K$-theory to algebraic geometry and number theory, Part I, II (Boulder, Colo., 1983), Amer. Math. Soc., Providence, RI, 1986, pp. 35-68.

[6] Spencer Bloch, The dilogarithm and extensions of Lie algebras, Algebraic K-theory, Evanston 1980 (Proc. Conf., Northwestern Univ., Evanston, Ill., 1980), Springer, Berlin, 1981, pp. 1-23.

[7] Spencer J. Bloch, Higher regulators, algebraic K-theory, and zeta functions of elliptic curves, CRM Monograph Series, vol. 11, American Mathematical Society, Providence, RI, 2000.

[8] Raoul Bott and Loring W. Tu, Differential forms in algebraic topology, Springer-Verlag, New York, 1982.

[9] J.-L. Brylinski and D. A. McLaughlin, The geometry of degree-4 characteristic classes and of line bundles on loop spaces. II, Duke Math. J. 83 (1996), no. 1, 105-139. 
[10] Jean-Luc Brylinski, Geometric construction of Quillen line bundles, Advances in geometry, Birkhäuser Boston, Boston, MA, 1999, pp. 107-146.

[11] P. Deligne, Le déterminant de la cohomologie, Current trends in arithmetical algebraic geometry (Arcata, Calif., 1985), Contemp. Math., vol. 67, Amer. Math. Soc., Providence, RI, 1987, pp. 93-177.

[12] _ Le symbole modéré, Inst. Hautes Études Sci. Publ. Math. (1991), no. 73, 147-181.

[13] Pierre Deligne, Théorie de Hodge. II, Inst. Hautes Études Sci. Publ. Math. (1971), no. 40, 5-57.

[14] Johan L. Dupont, The dilogarithm as a characteristic class for flat bundles, Proceedings of the Northwestern conference on cohomology of groups (Evanston, Ill., 1985), vol. 44, 1987, pp. 137-164.

[15] _ Scissors congruences, group homology and characteristic classes, Nankai Tracts in Mathematics, vol. 1, World Scientific Publishing Co. Inc., River Edge, NJ, 2001.

[16] Johan L. Dupont and Chih Han Sah, Scissors congruences. II, J. Pure Appl. Algebra 25 (1982), no. 2, 159-195.

[17] Hélène Esnault, Characteristic classes of flat bundles, Topology 27 (1988), no. 3, 323-352.

[18] Hélène Esnault and Eckart Viehweg, Deligne-Ben̆linson cohomology, Beullinson's conjectures on special values of $L$-functions, Academic Press, Boston, MA, 1988, pp. 43-91.

[19] Daniel Friedan and Stephen Shenker, The analytic geometry of two-dimensional conformal field theory, Nuclear Phys. B 281 (1987), no. 3-4, 509-545.

[20] Henri Gillet and Christophe Soulé, Arithmetic Chow groups and differential characters, Algebraic K-theory: connections with geometry and topology (Lake Louise, AB, 1987), NATO Adv. Sci. Inst. Ser. C Math. Phys. Sci., vol. 279, Kluwer Acad. Publ., Dordrecht, 1989, pp. 29-68.

[21] A. B. Goncharov, Explicit regulator maps on polylogarithmic motivic complexes, arXiv:math.AG/0003086.

[22] Phillip Griffiths and Joseph Harris, Principles of algebraic geometry, Wiley-Interscience [John Wiley \& Sons], New York, 1978, Pure and Applied Mathematics.

[23] Richard M. Hain, Classical polylogarithms, Motives (Seattle, WA, 1991), Proc. Sympos. Pure Math., vol. 55, Amer. Math. Soc., Providence, RI, 1994, pp. 3-42.

[24] Kirill Krasnov, Holography and Riemann surfaces, Adv. Theor. Math. Phys. 4 (2000), no. 4, 929-979, arXiv:hep-th/0005106.

[25] Serge Lang, Introduction to Arakelov theory, Springer-Verlag, New York, 1988.

[26] Katsuhiko Matsuzaki and Masahiko Taniguchi, Hyperbolic manifolds and Kleinian groups, Oxford Mathematical Monographs, The Clarendon Press Oxford University Press, New York, 1998, Oxford Science Publications.

[27] Henri Poincaré, Les fonctions fuchsiennes et l'équation $\Delta u=e^{u}$, J. Math. Pures Appl. 4 (1898), no. 5, 137-230.

[28] A. M. Polyakov, Quantum geometry of bosonic strings, Phys. Lett. B 103 (1981), 207-210.

[29] Dinakar Ramakrishnan, A regulator for curves via the Heisenberg group, Bull. Amer. Math. Soc. (N.S.) 5 (1981), no. 2, 191-195.

[30] Leon A. Takhtajan and Lee-Peng Teo, Liouville action and Weil-Petersson metric on deformation spaces, global Kleinian reciprocity and holography, arXiv:math.CV/0204318.

[31] Charles A. Weibel, An introduction to homological algebra, Cambridge University Press, Cambridge, 1994.

[32] P. G. Zograf, The Liouville action on moduli spaces, and uniformization of degenerating Riemann surfaces, Leningrad Math. J. 1 (1990), no. 4, 941-965.

[33] P. G. Zograf and L. A. Takhtadzhyan, On the Liouville equation, accessory parameters and the geometry of Teichmüller space for Riemann surfaces of genus 0, Mat. Sb. (N.S.) 132(174) (1987), no. 2, 147-166.

[34] - On the uniformization of Riemann surfaces and on the Weil-Petersson metric on the Teichmüller and Schottky spaces, Mat. Sb. (N.S.) 132(174) (1987), no. 3, 304-321, 444. 